\title{
Rapid cell-free characterization of multi-subunit CRISPR effectors and transposons
}

\author{
Franziska Wimmer $^{1 *}$, loannis Mougiakos ${ }^{1 *}$, Frank Englert ${ }^{1}$, Chase L. Beisel ${ }^{1,2 \#}$
}

${ }^{1}$ Helmholtz Institute for RNA-based Infection Research, Helmholtz Centre for Infection

Research, Würzburg, Germany

${ }^{2}$ Medical faculty, University of Würzburg, Würzburg, Germany

${ }^{*}$ Equal contributions

\#Correspondence to: chase.beisel@helmholtz-hiri.de (to C.L.B.) 


\section{ABSTRACT}

2 CRISPR-Cas biology and technologies have been largely shaped to-date by the characterization

3 and use of single-effector nucleases. In contrast, multi-subunit effectors dominate natural

4 systems, represent emerging technologies, and were recently associated with RNA-guided DNA

5 transposition. This disconnect stems from the challenge of working with multiple protein subunits

6 in vitro and in vivo. Here, we apply cell-free transcription-translation (TXTL) to radically accelerate

7 the characterization of multi-subunit CRISPR effectors and transposons. Numerous DNA

8 constructs can be combined in one TXTL reaction, yielding defined biomolecular readouts in

9 hours. Using TXTL, we mined phylogenetically diverse I-E effectors, interrogated extensively self-

10 targeting I-C and I-F systems, and elucidated targeting rules for I-B and I-F CRISPR transposons

11 using only DNA-binding components. We further recapitulated DNA transposition in TXTL, which

12 helped reveal a distinct branch of I-B CRISPR transposons. These capabilities will facilitate the

13 study and exploitation of the broad yet underexplored diversity of CRISPR-Cas systems and

14 transposons.

16 KEY WORDS

17 Cascade / CAST / PAM / PAM-DETECT / TXTL / Type I CRISPR-Cas system

19 HIGHLIGHTS

20 - PAM-DETECT for rapid determination of PAMs for Type I CRISPR-Cas systems in TXTL

21 - Mining of Type I orthologs and characterization of extensively self-targeting systems

22 - TXTL-based assessment of DNA target recognition and transposition by CRISPR

23 transposons

24 - Identification of a distinct branch of Type I-B CRISPR transposons 


\section{INTRODUCTION}

CRISPR-Cas systems endow prokaryotes with adaptive defense against invading elements and

possess effector nucleases that have become versatile biomolecular tools (Barrangou and

Doudna, 2016; Pickar-Oliver and Gersbach, 2019). These systems are remarkably diverse, with two classes, six types, over 30 subtypes, and a few subtype variants defined to-date (Makarova et al., 2019). The two classes are distinguished based on whether the effector nuclease responsible for CRISPR RNA (crRNA)-directed immune defense comprises a multi-protein complex (Class 1) or a single multi-domain protein (Class 2). While systems from both classes have undergone characterization, Class 2 systems have been the most extensively explored. For example, comprehensive determination of target-flanking protospacer-adjacent motifs (PAMs) (Leenay and Beisel, 2017) has been conducted for more than 100 Class 2 effectors spanning at least 15 subtypes (Collias and Beisel, 2021); in contrast, only for 10 Class 1 effectors spanning 7 subtypes (Table S1). This discrepancy belies the unique features of Class 1 systems that have attracted increasing attention for basic research and technology development (HidalgoCantabrana and Barrangou, 2020). Class 1 systems represent over $75 \%$ of all CRISPR-Cas

41 systems found in nature and contain phylogenetically diverse proteins possessing unique

42 mechanisms of action (Makarova et al., 2015). The associated machinery has also been recently

43 applied as tools in mammalian and plant cells, offering distinct means of achieving programmable

44 gene regulation and genome editing as well as the creation of variable chromosomal deletions

45 (Liu et al., 2018; Zheng et al., 2020). The same machinery has also been associated with 46 emerging alternative functions in bacteria, such as repressing expression of a toxin to promote

47 selection of the CRISPR-Cas system or to counter infection by phages encoding an inhibitory 48 anti-CRISPR protein (Acr) (Li et al., 2021). Finally, a subset of Class 1 systems contain Tn7-like

49 transposon genes and were shown to mediate crRNA-directed transposition (Klompe et al., 2019;

50 Petassi et al., 2020; Peters et al., 2017; Saito et al., 2021). These CRISPR transposons (CASTs)

51 have since been employed in bacteria for the efficient, programmable, and multiplexed insertion 
52 of donor DNA exceeding 10 kb (Klompe et al., 2019; Strecker et al., 2019; Vo et al., 2021). The

53 examples noted above highlight the potential of further exploring and harnessing Class 1

54 CRISPR-Cas systems and CASTs.

55 The disconnect between the broad relevance of Class 1 systems and the few well-

56 characterized examples can be largely attributed to the challenge of working with multiple protein

57 subunits. Cell-based assays are complicated by the need to encode and optimally express

58 multiple subunits from a minimal number of constructs, while in vitro assays require intensive

59 purification of multi-subunit complexes--tasks that are far simpler for single-effector nucleases. A

60 promising alternative came with the advent of cell-free transcription-translation (TXTL) systems

61 and their use for rapidly and scalably characterizing CRISPR-Cas systems (Garamella et al.,

62 2016; Jiao et al., 2021; Liao et al., 2019a, 2019b; Marshall et al., 2018; Maxwell et al., 2018;

63 Silverman et al., 2020; Watters et al., 2018). As part of a TXTL reaction, circular or linear DNA

64 constructs are added to the TXTL mix, resulting in the transcription and translation of the encoded

65 products in minutes to hours. Expressing CRISPR machinery targeted to an included reporter

66 construct further provides a quantitative and dynamic readout based on expression levels and

67 targeting activity. In our prior work, we showed that TXTL could functionally express the Type I

68 effector complex Cascade (CRISPR-associated complex for antiviral defense) that yielded

69 transcriptional repression of a reporter gene (Marshall et al., 2018). However, all other

70 implementations of TXTL to-date have focused on single-effector nucleases (Khakimzhan et al.,

71 2021; Liao et al., 2019a, 2019b; Wandera et al., 2020; Watters et al., 2018). Here, we leverage

72 TXTL to rapidly characterize diverse Type I systems and transposons, allowing ortholog mining,

73 characterization of self-targeting systems, and harnessing of CASTs. The resulting capabilities

74 are expected to accelerate the exploration and exploitation of this broad yet understudied branch

75 of CRISPR biology.

\section{RESULTS}


PAM-DETECT: a TXTL-based enrichment assay for PAM determination. One of the defining features of DNA-targeting CRISPR-Cas systems is the PAM (Leenay and Beisel, 2017). This collection of sequences always flanks a crRNA target and allows the effector nuclease to discriminate between self (the equivalent targeting spacer in the CRISPR array) and non-self (the invader). However, the associated sequences can vary widely even between close homologs (Collias and Beisel, 2021). Given that the comprehensive PAM determination assays applied for Class 1 systems involved laborious in vitro or cell-based assays (Table S1), we devised a TXTLbased assay that could elucidate the complete PAM profile recognized by an effector complex but without the need for protein purification or cellular expression (Figs. 1A and B). The assay involves expressing the crRNA and the three to five Cas proteins that form Cascade, which then binds target DNA. While Cascade binding normally recruits the endonuclease Cas3 to nick and processively degrade the non-target strand of DNA (Huo et al., 2014; Mulepati and Bailey, 2013; Westra et al., 2012), Cascade strongly binds DNA even without Cas3 (Jore et al., 2011; Westra et al., 2012). As part of the TXTL-based assay, Cascade binds target DNA flanked by a library of potential PAM sequences. After sufficient time to produce Cascade and ensure DNA binding, a restriction enzyme is introduced that cleaves a sequence within the DNA target. As a result, DNA containing a recognized PAM sequence is protected by the bound Cascade, thereby enriching

95 this sequence within the library. Next-generation sequencing (NGS) is then performed to quantify 96 the relative frequency of each PAM sequence before and after restriction digestion. We call this 97 assay PAM-DETECT (PAM-DETermination with Enrichment-based Cell-free TXTL). From the 98 addition of the DNA constructs to the isolation of library DNA for NGS, the entire process requires 9913 to 23 hours -- substantially faster than the days to weeks required for in vitro and cell-based 100 assays when starting with DNA expression constructs. Also, because the reactions are conducted 101 in a few microliters, reactions can be conducted in parallel in microtiter plates for characterizing a 102 massive number of systems and conditions at one time. 
As part of PAM-DETECT, we devised two parallel checkpoints to assess the extent of library protection and PAM enrichment prior to submitting samples for NGS. For the first checkpoint (Fig. 1C), qPCR is applied with a digested and undigested library to measure the extent to which the library was protected by Cascade binding. Given that excess effector can boost the prevalence of less-preferred PAM sequences (Karvelis et al., 2015), the qPCR results can indicate the stringency of the determined PAM sequences. Fortunately, the conditions of

109 PAM-DETECT can be readily tuned by changing the concentration of the added DNA constructs 110 and the time allowed for Cascade expression and DNA binding. For the second checkpoint (Fig.

111 1D), the digested and undigested libraries are subjected to Sanger sequencing. Elevated peaks

112 in the digested sample reflect enrichment of those bases at that PAM position, providing an early 113 indication of the determined PAM.

PAM-DETECT validated with the canonical Type I-E CRISPR-Cas system from Escherichia coli. To evaluate PAM-DETECT, we began with Cascade encoded by the Type I-E CRISPR-Cas system from Escherichia coli (Fig. 2A), the best studied Type I system to-date. As part of its extensive characterization, the effector complex has been subjected to multiple comprehensive PAM determination assays (Caliando and Voigt, 2015; Fineran et al., 2014; Fu et al., 2017; Leenay et al., 2016; Musharova et al., 2019; Xue et al., 2015), establishing a complex landscape

121 principally composed of the canonical PAM sequences AAG, AGG, ATG, and GAG (written 5' to $1223^{\prime}$ ) located on the non-target strand immediately upstream of the guide sequence. We applied 123 PAM-DETECT by encoding the five Cascade genes and a targeting single-spacer CRISPR array 124 encoding a crRNA on six separate plasmids and combining these plasmids with a 5-base PAM 125 target library in TXTL (Fig. 2A). To explicitly evaluate the impact of excess effector complexes, 126 we tested two different conditions: one with $0.25 \mathrm{nM}$ of Cascade-encoding plasmids and 6-hour 127 reaction time for low Cascade expression and binding, and another with $3 \mathrm{nM}$ of Cascade128 encoding plasmids and 16-hour reaction time for high Cascade expression and binding. The 
129 intermediate qPCR check showed significant DNA protection compared to the control lacking

130 Cascade, with $\sim 2$-fold more protection with the high versus low Cascade condition (Fig. 2B).

131 Correspondingly, the Sanger sequencing checkpoint showed enrichment of an AAG motif

132 compared to the undigested control, where the motif was more pronounced for the low Cascade

133 condition (Fig. 2C). The checkpoints were in line with protection of DNA sequences related to the

134 known PAM, with enhanced protection for the high Cascade condition.

135 Given the promising results from the two checkpoints, we proceeded to NGS with both

136 Cascade conditions to map the full PAM profile. After determining an enrichment score for each

137 library sequence, we visualized the results as a PAM wheel to capture both individual sequences

138 and enrichment scores (Leenay et al., 2016) (Fig. 2D). The PAM wheel for the low Cascade

139 condition captured the four known canonical PAMs as well as other well-recognized PAM

140 sequences (e.g. TAG, AAC) reported in prior screens (Caliando and Voigt, 2015; Fineran et al.,

141 2014; Leenay et al., 2016; Musharova et al., 2019; Xue et al., 2015). The PAM wheel for the high

142 Cascade condition included these PAM sequences as well as other PAM sequences that were

143 less enriched (e.g. AAA, AAT) or negligibly enriched (e.g. CAG, ATT) for the low Cascade

144 condition (Fig. 2D). The differences in PAM profiles demonstrate how PAM-DETECT can be

145 readily tuned by varying plasmid concentration and reaction time.

146 To validate the results, we applied TXTL to silence expression of a deGFP reporter (Shin

147 and Noireaux, 2012) using a distinct target sequence overlapping the reporter's upstream

148 promoter (Fig. 2E, Table S2). The PAM region could then be altered without affecting the

149 promoter sequence. For representative PAM sequences, the fold-repression of deGFP production

150 versus a non-targeting control strongly correlated with the enrichment score of each sequence in

151 PAM-DETECT for the low Cascade condition $\left(\mathrm{R}^{2}=0.99\right)$ (Fig. 2F). The correlation was

152 particularly striking given the use of a different target sequence, which can affect the apparent

153 hierarchy of PAM recognition (Leenay et al., 2016; Xue et al., 2015). Applying the same assay to

154 PAM sequences enriched under the high Cascade condition but not detected with our previous 
155 PAM-SCANR method (Leenay et al., 2016), we measured modest but significant deGFP

156 repression (Fig. 2G). These validation experiments show that PAM-DETECT can produce 157 comprehensive and quantitative PAM profiles, and the assay conditions can be readily altered to 158 tune the stringency of PAM detection.

Distinct PAM profiles pervade I-E CRISPR-Cas systems. After validating PAM-DETECT using

161 the established I-E system from E. coli, we turned to the first important use of this assay: mining 162 diverse CRISPR effector proteins and complexes. Nuclease mining has been highly successful 163 for single-effector nucleases such as Cas9, which revealed a wide collection of nucleases 164 recognizing the full spectrum of PAMs (Gasiunas et al., 2020; Zetsche et al., 2020). Nuclease 165 mining therefore could be highly valuable when applied to Class 1 systems. Focusing again on 166 the I-E subtype of CRISPR-Cas systems, we began by identifying diverse Cas8e proteins 167 responsible for PAM recognition within Cascade from known cultured mesophilic bacterial strains. 168 This analysis revealed a set of 213 Cas8e proteins (Table S3). We further divided the Cas8e set 169 in groups according to the amino-acid sequence of the highly variable L1 loop within the $\mathrm{N}$ 170 terminal domain (Table S3) reported to stabilize the Cas8e-PAM interactions (Tay et al., 2015; 171 Xiao et al., 2017). The numerous clusters with distinct L1 motifs suggested diverse modes of PAM 172 recognition extending beyond that observed with E. coli's Cascade.

173 We selected 11 representative I-E systems reflecting some of the most abundant L1 motifs 174 to characterize with PAM-DETECT (Figs. 3A, S1). Characterizing the resulting Cascade 175 complexes required encoding 55 Cascade genes and 11 single-spacer arrays, each in separate 176 plasmids. However, despite this large number of constructs, PAM-DETECT could be performed 177 with all constructs in parallel. We selected the high Cascade conditions ( $3 \mathrm{nM}$ plasmids, 16 hour 178 reaction time) given uncertainty about how well a given system would be functionally expressed 179 in TXTL. All but one system yielded significant enrichment of the PAM library compared to a non180 digested control (Fig. S1A), allowing us to determine a large number of PAM profiles. 

profile most distinct from that associated with the E. coli Cascade was recognized by Cascade from Streptococcus thermophilus DGCC 7710 (Sth), which recognized any sequence with an A or $\mathrm{T}$ at the -1 position as well as $\mathrm{AS}(\mathrm{S}=\mathrm{G}, \mathrm{C})$ and ATS. While the $\mathrm{S}$. thermophilus Cascade protected $\sim 75 \%$ of the library -- indicative of enriched sub-optimal PAMs, the PAM profile matched the few PAM sequences previously confirmed to bind purified Cascade in vitro (Sinkunas et al., 2013). Most remaining systems generally recognized AAG as a dominant PAM sequence, 188 although there were notable deviations and additions. For example, one system from Azotobacter 189 chroococcum NCIMB 8003 (Ac2) principally recognized AA, while another system from 190 Paracoccus sp. J4 (Ps) preferentially recognized AAC. Separately, the systems from Marinomonas sp. MWYL1 (Ms), and Ectothiorhodospira haloalkaliphila ATCC 51935 (Eh) as well as a separate system in Azotobacter chroococcum NCIMB 8003 (Ac3) recognized PAM profiles paralleling that recognized by E. coli's system. Notably, Ac2 and Ac3 are present in the same bacterium, suggesting that their partially overlapping PAM profiles could confer redundancy in immune defense as reported for co-occurring Type I and Type III systems (Silas et al., 2017). The distinct PAM profiles that gave measurable activity in the deGFP silencing assay in TXTL confirmed the trends observed with the PAM wheels (Figs. 3B). Given that Type I-E systems represent one of the most abundant CRISPR-Cas subtypes in nature (Makarova et al., 2015), our initial characterization suggests that a far greater diversity of recognized PAM profiles likely exists across this expansive subtype. are functionally encoded. Beyond mining individual systems, PAM-DETECT can be further applied to interrogate systems that deviate from traditional immune defense. Prominent examples are self-targeting CRISPR-Cas systems that encode crRNAs targeting chromosomal locations 206 (Wimmer and Beisel, 2019). While self-targeting is considered inherently incompatible with a 
207 functional CRISPR-Cas system (Gomaa et al., 2014; Stern et al., 2010; Vercoe et al., 2013),

208 accumulating examples provide important counterpoints where the systems tolerate or even

209 utilize self-targeting crRNAs. For instance, systems encoding self-targeting crRNAs have been

210 associated with prophage-encoded Acrs that actively repress immune defense and serve as

211 markers to uncover novel Acrs (Marino et al., 2018; Rauch et al., 2017; Watters et al., 2018; Yin

212 et al., 2019). Furthermore, a crRNA-like RNA encoded within Type I systems was also shown to

213 direct Cascade to a partially complementary site upstream of a toxin gene, thereby blocking its

214 transcription to ensure maintenance of the CRISPR-Cas system and counter Acr-encoding

215 phages (Li et al., 2021). PAM-DETECT and TXTL therefore could accelerate the characterization

216 of these unique systems.

We specifically focused on two extensively self-targeting CRISPR-Cas systems within the 218 plant pathogen Xanthomonas albilineans CFBP7063. This bacterium encodes two CRISPR-Cas 219 systems (I-C and I-F1) each harboring the full cohort of cas genes and associated with a remarkably large repertoire of self-targeting spacers (Fig. 4A). Of the 64 spacers present across

221 the six CRISPR arrays, $24(38 \%)$ at least partially match sites in the chromosome or one plasmid 222 (Table S4, Fig. S2A) with a common set of flanking PAMs (Fig. 4B). TXTL therefore offered a 223 rapid means to explore the functionality of these systems and why self-targeting is tolerated.

We first performed PAM-DETECT using Cascade from both CRISPR-Cas systems (Fig.

225 4C). Either Cascade protected a small portion of the DNA library ( 2\% for I-C, $\sim 6 \%$ for I-F1) from

226 restriction digestion (Fig. S2B), indicating functional expression of all Cascade subunits. PAM-

227 DETECT further revealed PAM profiles that overlapped -- but were not identical to -- the I-C and

228 I-F1 systems with even a moderately mapped PAM profile (Almendros et al., 2012; Leenay et al.,

229 2016; Rao et al., 2017; Rollins et al., 2015; Tuminauskaite et al., 2020; Zheng et al., 2019). In

230 particular, the I-C system from $X$. albilineans recognizes TTC followed by TTT and CTC, while the

231 characterized I-C system from Bacillus halodurans recognizes TTC followed by CTC and then

232 TCC (Leenay et al., 2016) and the I-C system from Legionella pneumophila recognized TTC 
233 followed by TTT and CTT (Rao et al., 2017). Separately, the I-F1 system from X. albilineans 234 recognizes CC as the strongest PAM similar to other I-F systems (Almendros et al., 2012; Rollins 235 et al., 2015; Tuminauskaite et al., 2020; Zheng et al., 2019), although $X$. albilineans system also 236 can recognize a $\mathrm{G}$ and $\mathrm{T}$ but not an $\mathrm{A}$ at the -2 position and could tolerate a CC PAM shifted one 237 nucleotide upstream. The recognized PAMs of both I-C and I-F1 systems further overlapped with 238 the PAM sequences flanking the self-targets for $87 \%$ of the I-C self-targets (TTC, TTT, CTC) and 239 all I-F1 self-targets (CC, CCT) (Figs. 4B and C). Testing these individual PAMs in TXTL using 240 gene repression with Cascade confirmed that the I-C system could recognize not only TTC but 241 also TTT and CTC (Fig. 4D). The same TXTL assay confirmed that the I-F1 system could 242 recognize the CC PAM associated with almost all self-targeting. PAM-DETECT therefore can be 243 implemented beyond I-E systems and indicated that the interrogated I-C and I-F1 systems in $X$. albilineans are capable of binding the vast majority of self-targeting sites in the genome. recognition of these self-targeting sites should prove lethal to this bacterium. We therefore reconfigured the TXTL assay to evaluate the extent to which the I-C or I-F1 Cas3 could elicit DNA degradation (Fig. 4E). The DNA target was placed in the backbone of the deGFP reporter $\sim 200$ bps upstream of the deGFP promoter flanked by a TTC (I-C) or CC (I-F1) PAM, which would only lead to loss of deGFP fluorescence if the backbone is nicked or cleaved, leading to DNA degradation by RecBCD (Marshall et al., 2018). For both systems, this new target site location resulted in targeted deGFP silencing following expression of Cascade and Cas3 but not Cascade

253 alone (Fig. 4E). Cas3 is therefore functionally encoded and would lead to lethal self-targeting 254 unless Cascade is fully silenced in this bacterium or another mechanism is in place to inhibit 255 Cascade and/or Cas3 activity. The findings thus lay a foundation to investigate the mechanistic 256 basis of self-targeting and whether self-targeting underlies functions extending beyond immune 257 defense. 

created a unique opportunity: applying the same assay to CASTs. Of the three known CAST types (I-B, I-F, V-K), two (I-B, I-F) rely on Cascade for DNA target recognition (Klompe et al., 2019; Saito et al., 2021). Recognition then leads to integration of the transposon DNA at a defined distance downstream of the target. Characterization of these systems to-date has relied on encoding a crRNA, all CRISPR and transposon components, and donor DNA flanked by the transposon ends in bacteria to achieve targeted transposition. However, the reliance of I-B and I-

267 F CASTs on Cascade offers an opportunity to express only these CAST components as part of 268 PAM-DETECT to elucidate key rules for DNA target recognition.

We began with the I-F CAST from $V$. cholerae that exhibited robust DNA integration in $E$. coli and has been used for multiple applications in bacteria (Klompe et al., 2019; Vo et al., 2021) (Fig. 5A). Prior screening of individual potential PAM sequences via transposition in E. coli revealed a general preference for a $\mathrm{C}$ at the -2 position, although a comprehensive PAM remained

273 to be determined. We therefore applied PAM-DETECT by expressing the three Cascade genes

274 (a natural cas8-cas5 fusion, cas6, and cas7) along with the tniQ gene responsible for recruiting 275 the other three transposon genes ( $\operatorname{tns} A, \operatorname{tns} B, \operatorname{tns} C)$, as the role of TniQ in DNA target recognition 276 remained to be established (Klompe et al., 2019; Petassi et al., 2020; Vo et al., 2021). PAM277 DETECT revealed 57\% DNA protection under high Cascade conditions (3 nM plasmids, 16 hour 278 reaction time), leading us to also perform PAM-DETECT with the low Cascade conditions (0.25 279 nM plasmids, 6 hour reaction time) that exhibited 25\% DNA protection (Fig. S3A). We further 280 found that tniQ was dispensable for DNA binding (Fig. S3B). The resulting PAM profile was 281 remarkably flexible, with a preference for a $\mathrm{C}$ and bias against an A the -2 position (Figs. 5B, 282 S3C). We further noticed deviations from these biases that could still allow target recognition. For 283 example, recognition of a $\mathrm{G}$ or $\mathrm{T}$ at the -2 position could be enhanced with a $\mathrm{C}$ at the -1 position 284 or an $\mathrm{A}$ at the -3 position. Separately, an $\mathrm{A}$ at the -2 position could be rescued with a $\mathrm{C}$ at the -3 
position (Figs. 5B, S3C). The results from PAM-DETECT therefore suggest that this I-F CAST recognizes a remarkably flexible PAM profile with preferences extending beyond a simple consensus sequence.

To evaluate the PAM profile output by PAM-DETECT, we first employed our TXTL-based deGFP silencing assay (Figs. 5C). Cascade most strongly recognized PAM sequences with $\mathrm{C}$ at

290 the -2 position, with the greatest preference for CC. Deviating from this preference reduced but 291 did not eliminate measurable silencing as long as A was not present at the -2 and -3 positions. 292 Interestingly, while AAA and AAT yielded no measurable deGFP silencing, replacing A with C at 293 the -3 position restored measurable silencing, albeit with low activity (Fig. 5D). These small but 294 measurable differences raised the question of how these activities translate into programmable 295 DNA transposition in E. coli. We therefore employed the previously described transposition system in which the CAST genes and crRNA are encoded outside of donor DNA flanked by the transposition ends (Klompe et al., 2019), and transposition is conducted at $30^{\circ} \mathrm{C}$ for higher integration efficiency (Vo et al., 2021). The crRNA is further designed to drive transposition into the lacZ gene in the $E$. coli genome, which yields white rather than blue colonies on the cleavable yielded robust DNA transposition, even though the targets were separated by only one base

(Figs. 5E, S3D and E). Furthermore, the measured transposition efficiency was similar for CAA and CC. Therefore, even low levels of gene silencing with Cascade in TXTL could yield efficient transposition in E. coli.

307 profile. Building on our success applying PAM-DETECT to the I-F CAST from V. cholerae, we 308 turned to I-B CASTs. Two examples of I-B CASTs were experimentally characterized very 309 recently, revealing that a second encoded tniQ (renamed $t n s D$ ) drives DNA transposition at 310 conserved sites flanking tRNAs or glmS independently of Cascade or a crRNA (Saito et al., 2021). 
311 These examples were also previously subjected to a high-throughput PAM determination assay

312 conducted by performing transposition in vivo expressing all components in E. coli. Type I-B

313 CASTs were further split into two subtypes (I-B1, I-B2) based on the TnsA and TnsB being fused

314 or separate proteins, the general genetic organization of the CAST locus, and crRNA-independent

315 insertion flanking tRNAs or glmS.

316 While exploring examples within the I-B CASTs, we noticed a further division within the I-

317 B2 subtype typified by tns $D$ flanking the Cascade genes rather than the other transposon genes

318 (Fig. 6A). This organization more closely paralleled that of I-B1 CASTs (Saito et al., 2021) but still

319 possesses the tnsAB fusion and the presence of tRNAs flanking the CASTs indicative of I-B2

320 CASTs. The division of the I-B2 CASTs in two clades, denoted hereafter as I-B2.1 and I-B2.2,

321 was further supported by the higher shared similarity of the TnsAB, TnsC, TnsD and TniQ proteins

322 from systems that belong to each clade (Figs. 6A, S4A). The Cascade proteins were similar

323 across all I-B CASTs and thus could not help differentiate any divisions within this CAST type

324 (Saito et al., 2021). We chose the I-B2.2 CAST from Rippkaea orientalis (RoCAST) as a

325 representative example to characterize.

We conducted PAM-DETECT by expressing a single-spacer CRISPR array as well as the

327 four RoCAST Cascade genes (cas5, cas6, cas7, cas8) from two separate expression constructs.

328 This combination yielded a PAM profile dominated by ATG (Figs. 6B, S4B and C), matching the

329 PAM recognized by the one previously characterized I-B2.1 CAST from Peltigera membranacea

330 cyanobiont 210A (PmcCAST) (Saito et al., 2021). This match was expected given the high

331 similarity (65-81\%) between the protein components forming PmcCAST and RoCAST Cascade.

332 However, single-nucleotide perturbations to ATG could be recognized by the RoCAST even under

333 low Cascade conditions. The TXTL-based deGFP silencing assay confirmed recognition of ATG

334 as well as the single-nucleotide perturbations (Fig. 6C). We further showed that PAM-DETECT

335 can be applied to the previously characterized I-B1 CRISPR transposon from Anabaena variabilis 
ATCC 29413 (AvCAST) (Saito et al., 2021) (Fig. S5A and B). These insights came from using a streamlined TXTL assay without any protein or RNA purification and only half of the genetic components needed for transposition.

DNA transposition by CRISPR transposons can be recapitulated in TXTL. We next wanted

341 to evaluate how insights into PAM recognition translate into DNA transposition. However, doing

342 so with in vitro or cell-based assays posed numerous challenges that would slow the

343 characterization process. In particular, encoding and expressing all of the genetic components

344 into a few compatible plasmids is laborious and could require extensive optimization, while

345 overexpressing some components could be toxic to the cells. Instead, we asked whether

346 transposition could be recapitulated in TXTL (Fig. 7A) to rapidly test different configurations and

347 constructs.

We began with the $V$. cholerae I-F CAST. Combining DNA constructs encoding a targeting

349 single-spacer array, three Cascade genes, four transposon genes (tns $A, \operatorname{tns} B, \operatorname{tns} C$, tniQ), donor

350 DNA flanked by the transposon ends, and a target construct resulted in measurable DNA

351 transposition in both orientations by PCR (Fig. S6A). Sanger sequencing of the PCR products

352 revealed the core transposon ends as well as the distance between the target site and insertion

353 site that aligned with prior work (Fig. S6A). We were also able to reconstitute transposition in

354 TXTL for AvCAST (Fig. S5C). Therefore, TXTL can be used to characterize DNA transposition 355 by CASTs.

DNA transposition in TXTL with the Rippkaea orientalis CAST establishes a distinct branch

358 within I-B2 CRISPR transposons. Building on TXTL-based transposition with the I-F and I-B2.1

359 CASTs, we evaluated DNA transposition in TXTL with the I-B2.2 RoCAST (Fig. 7B). Because the 360 ends of this transposon were unclear, we constructed a donor DNA construct flanked by two 250361 bp sequences predicted to contain the right and left RoCAST ends. We combined the donor DNA 
and target DNA flanked by an ATG PAM with constructs encoding the I-B2.2 Cascade genes

363 (cas5, cas6, cas7, cas8), transposase genes (tns $A B$, tns $C$, tnsD, tniQ), and a single-spacer

364 CRISPR array with a targeting or non-targeting spacer. The TXTL reactions resulted in

365 measurable crRNA-directed transposition in both orientations by PCR. Sanger sequencing of the

366 PCR products revealed the core transposon ends along with five bases that are duplicated as

367 part of transposition (Fig. 7B), similar to other CASTs (Klompe et al., 2019).

368 Recent work revealed that I-B CASTs possess two distinct modes of transposition:

369 CRISPR-dependent transposition through TniQ and DNA targeting by Cascade and CRISPR-

370 independent transposition through TnsD (Saito et al., 2021). We therefore evaluated the role of

371 TniQ and TnsD for either mode of transposition in TXTL. For CRISPR-dependent transposition,

372 TXTL reactions with TniQ yielded the highest CRISPR-dependent transposition efficiency.

373 However, we surprisingly observed modest but detectable crRNA-dependent transposition even

374 in the absence of TniQ and TnsD by PCR (Fig. 7B and C) and by next-generation sequencing of

375 the PCR product (Fig. S6B). As further support for I-B2.2 as a separate branch, TniQ was

376 reported to be required for crRNA-dependent transposition by the I-B1 AvCAST (Fig. S5C) and

377 the I-B2.1 PmcCAST (Saito et al., 2021). To explore CRISPR-independent transposition, we

378 swapped the crRNA target for the tRNA-Leu gene naturally flanking RoCAST in the $R$. orientalis

379 genome. CRISPR-independent transposition was detected in both orientations (Fig. 7D).

380 Transposition required TnsAB, TnsC and TnsD, while removing TnsD or replacing it with TniQ

381 eliminated transposition.

We finally asked how the properties of RoCAST observed in TXTL translate in vivo. We

383 adapted the DNA constructs for use in E. coli by condensing the constructs into three plasmids

384 (Fig. 7E and F). For CRISPR-dependent transposition, we targeted the lacZ gene in the E. coli

385 genome at a site flanked by an ATG PAM. Over-expressing Cascade proved to be cytotoxic,

386 reflecting challenges to characterizing CASTs in vivo, although the cytotoxicity could be relieved

387 with minimal induction of Cascade expression. In line with the TXTL results, CRISPR-dependent 
transposition was measurable by PCR in E. coli strains expressing the Cascade, TnsAB, TnsC and TniQ proteins, albeit only for the left-to-right insertion orientation (Fig. 7E). Removing TnsD boosted this mode of transposition (Fig. 7E). Somewhat paralleling the TXTL results, less efficient transposition was measurable by PCR in the absence of TniQ but not both TniQ and TnsD (Figs.

7E and S6C). For CRISPR-independent transposition, we targeted a vector carrying the terminal

393 region of the tRNA-Leu gene from the $R$. orientalis genome. Matching the TXTL results, TnsAB,

394 TnsC, and TnsD proteins were necessary for transposition (Fig. 7F). To compare the insertion 395 distances between the target and the inserted donor DNA in TXTL and in E. coli, the PCR products 396 were subjected to next-generation sequencing. For CRISPR-dependent transposition, 397 transposition in TXTL consistently occurred 78 bps downstream of the PAM, while transposition 398 in E. coli principally occurred within a window of 83-89 bps downstream of the PAM (Fig. 7G), 399 although the difference may be attributed to the use of different target sites and insertion contexts 400 as was previously reported for the I-B1 AvCAST (Saito et al., 2021). For CRISPR-independent 401 transposition, transposition in TXTL and in E. coli both occurred 31 bps downstream of the tRNA402 Leu gene (Fig. 7H). The insertion distances for both modes of transposition are comparable to 403 the insertion windows identified for the other characterized I-B2 system (Saito et al., 2021). 404 Overall, these findings demonstrate that insights from TXTL-based transposition translate into in 405 vivo settings.

\section{DISCUSSION}

408 Through multiple demonstrations, we showed how cell-free TXTL reactions could be applied to 409 rapidly characterize multi-component CRISPR nucleases as well as CRISPR transposons. One 410 method we used repeatedly, PAM-DETECT, could comprehensively determine PAM sequences 411 recognized by the DNA-binding machinery of an immune system or transposon. Our method 412 offered important advantages over current cell-based and in vitro-based methods that should 413 accelerate characterization of Class 1 CRISPR-Cas systems and transposons. PAM-DETECT 
414 could be completed in under one day starting from purified DNA constructs and ending with

415 amplicons for next-generation sequencing. In contrast, cell-based methods require DNA

416 transformation, culturing, and growth before DNA isolation that can stretch for days. In vitro

417 assays can require even more time due to the need to purify ribonucleoprotein complexes

418 overexpressed in cells. Both traditional methods can require extensive optimization, such as

419 combining the constructs into a small set of compatible plasmids with appropriate expression,

420 tackling issues of toxicity, or troubleshooting issues that arise during purification--steps that are

421 irrelevant for TXTL. Finally, the ability to conduct reactions in a few microliters allows PAM-

422 DETECT to be readily scaled, allowing the parallel interrogation of tens or even hundreds of

423 systems under different reaction conditions. While TXTL reactions are normally conducted

424 between $25^{\circ} \mathrm{C}$ and $37^{\circ} \mathrm{C}$, the DNA-binding and restriction steps could be conducted at elevated

425 temperatures, such as for evaluating CRISPR-Cas systems derived from thermophiles and

426 hyperthermophiles. In addition, while overexpression of Cascade could lead to unwanted

427 enrichment of suboptimal PAMs, we demonstrated how the reaction conditions could be tuned

428 and how qPCR could be applied to gauge the extent of library protection. Given these advantages,

429 TXTL-based characterization of Class 1 systems could represent a widespread means to explore

430 these abundant and diverse systems.

431 We further leveraged TXTL to accelerate the validation and extension of our results from

432 PAM-DETECT. We frequently employed a deGFP repression assay in which target binding by

433 Cascade blocks deGFP expression. This assay allowed us to confirm PAM sequences, where

434 deGFP repression strongly correlated with enrichment with PAM-DETECT for the E. coli I-E

435 system. One potential limitation to PAM-DETECT and the repression assay is that binding may

436 not correspond to DNA degradation, as was reported to some degree for DNA binding and

437 degradation by the I-E system (Xue et al., 2015). However, as part of characterizing the self-

438 targeting CRISPR-Cas systems in $X$. albilineans, we showed that the repression assay could be

439 readily modified to specifically assess DNA degradation by Cas3. By targeting a location well 
440 upstream of the promoter, a reduction of deGFP expression would only occur through the action

441 of Cas3. This altered setup could be readily applied to validate identified PAMs in the context of

442 DNA degradation. Finally, we showed that DNA transposition by CASTs could be fully

443 recapitulated in TXTL. We were able to recapitulate CRISPR-dependent and CRISPR-

444 independent transposition by I-B and I-F CASTs, suggesting that TXTL would be valid for V-K

445 CASTs representing the third and final subtype (Saito et al., 2021; Strecker et al., 2019). With

446 these additional assays in place, TXTL can be applied well beyond PAM determination.

447 One major application we pursued was mining the natural diversity of I-E CRISPR-Cas

448 systems. Using PAM-DETECT, we evaluated 11 different systems representing diverse

449 sequences within the variable L1 loop of the Cas8e protein. The analysis revealed ranging extents

450 of library protection indicative of Cascade expression, binding activity, and the breadth of

451 recognized PAMs. The identified PAMs deviated from that associated with E. colis I-E system,

452 suggesting that a far broader range of PAMs could be revealed by further interrogating the

453 diversity of these systems. Whether the diversity parallels that observed for Cas 9 nucleases

454 remains to be seen and could reflect the distinct forces that shaped the evolution of each system

455 type (Gasiunas et al., 2020). A similar approach could be particularly powerful for mining I-C and

456 I-Fv Cascade complexes that require the fewest number of Cas proteins (Hochstrasser et al.,

457 2016; Pausch et al., 2017). Complexes could be mined exhibiting not only unique PAM

458 preferences but also smaller proteins, altered temperature ranges, or enhanced binding and

459 cleavage activities. Given the proliferation of engineered single-effectors with altered PAM

460 recognition (Collias and Beisel, 2021), TXTL could be applied to characterize any similarly

461 engineered variants of type I systems.

462 Beyond mining orthologs within a CRISPR-Cas subtype, PAM-DETECT offered a powerful

463 means to interrogate CRISPR-Cas systems with potentially unique properties. We specifically

464 focused on a I-C system and a I-F1 system present in $X$. albilineans that encode a large repertoire

465 of self-targeting spacers. While genetic deactivation of the CRISPR machinery is thought to be a 
common means of resolving otherwise lethal self-targeting (Stern et al., 2010), we showed that

467 Cascade and Cas3 were functionally encoded and could recognize PAMs flanking the vast 468 majority of the self targets. These findings instead suggest that the expression or activity of the 469 CRISPR machinery is inhibited, preventing lethal self-targeting. One possibility is that the cell 470 encodes Acrs that actively inhibit steps of CRISPR-based immunity or expression (Davidson et 471 al., 2020). Future work therefore could interrogate what is preventing both systems from lethal 472 self-targeting not only in $X$. albilineans but also the many other organisms possessing CRISPR473 Cas systems with self-targeting spacers. This work could reveal novel classes of Acrs as well as 474 instances of CRISPR-Cas systems performing functions extending beyond adaptive immunity.

475 As a final example, we applied TXTL to characterize a distinct branch of I-B2 CASTs. The

476 I-B CAST type was recently divided into two subtypes (I-B1 and I-B2) based on whether tnsA and

477 tnsB were fused, the genetic organization of the CAST, and the site recognized for CRISPR478 independent insertion (Saito et al., 2021). When exploring I-B2 CASTs, we noticed a clear division 479 in the genetic organization of these CASTs that paralleled phylogenetic trees for the transposon 480 genes. We further found that CRISPR-dependent transposition could occur in the absence of 481 TniQ for one branch (I-B2.2), contrasting with the essential role of TniQ described for the other 482 branch (I-B2.1) and subtype (I-B1) (Saito et al., 2021). TniQ-independent transposition under 483 these conditions was weak, raising questions whether CRISPR-dependent transposition would 484 occur in the absence of TniQ under natural settings. Regardless of the biological relevance, it 485 likely reflects distinct biomolecular mechanisms and interactions that further support some 486 division in categorization. As only a small number of CASTs have been characterized to-date, 487 further exploring these unique mobile genetic elements could reveal new properties and provide 488 CASTs for further technological development and application. In that regard, applying cell-free 489 systems could greatly aid these efforts and help drive new discoveries and technologies. 


\section{ACKNOWLEDGEMENTS}

492 We thank Natalia Ivanova for assistance with the bioinformatics identification of Type I-E CRISPR-

493 Cas systems. We thank Sam Sternberg for providing plasmid pSL0283, pSL0284 and pSL0527.

494 This work was supported by an ERC Consolidator grant (865973 to C.L.B.), the Deutsche

495 Forschungsgemeinschaft (BE 6703/1-1 to C.L.B.), and the Netherlands Organization for Scientific

496 Research (NWO) through a Rubicon Grant (project 019.193EN.032 to I.M.). A portion of this

497 research was performed under the JGI-EMSL Collaborative Science Initiative and used resources

498 at the DOE Joint Genome Institute and the Environmental Molecular Science Laboratory, which

499 are DOE Office of Science User Facilities. Both facilities are sponsored by the Office of Biological

500 and Environmental Research and operated under Contract Nos. DE-AC02-05CH11231 (JGI) and

501 DE-AC05-76RL01830 (EMSL).

502

503 AUTHOR CONTRIBUTIONS

504 Conceptualization: F.W., I.M., C.L.B.; Methodology: F.W., I.M., C.L.B., Software: F.W., I.M., 505 Validation: F.W., I.M., F.E.; Investigation: F.W., I.M., F.E., Writing - Original Draft: F.W., I.M., 506 C.L.B., Writing - Review \& Editing: F.W., I.M., F.E., C.L.B. Visualization: F.W., I.M., C.L.B., 507 Supervision: C.L.B.; Funding acquisition: C.L.B.

508

509 DECLARATION OF INTERESTS

510 C.L.B. is a co-founder and member of the Scientific Advisory Board member for Locus 511 Biosciences and is a member of the Scientific Advisory Board for Benson Hill. The other authors 512 declare no competing interests. 


\section{FIGURE TITLES AND LEGENDS}

514
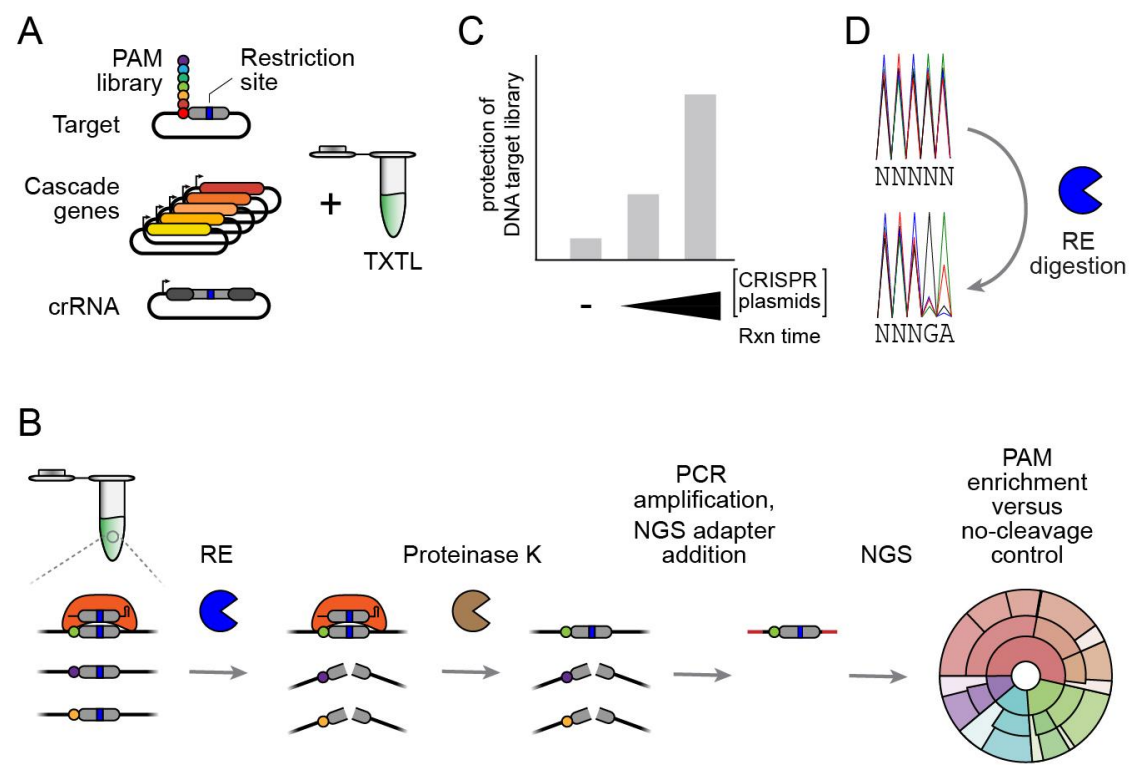

516 Figure 1. PAM-DETECT, a TXTL-based PAM determination assay for multi-protein CRISPR

517 effectors.

518 (A) DNA components added to a TXTL reaction to perform PAM-DETECT. The Cascade genes

519 can be encoded on separate plasmids as shown here or as an operon.

520 (B) Steps comprising PAM-DETECT. RE: restriction enzyme.

521 (C) Determination of library protection from restriction cleavage by qPCR. A reaction conducted

522 without the Cascade and crRNA plasmids serves as a negative control.

523 (D) Determination of PAM enrichment by Sanger sequencing. 
A
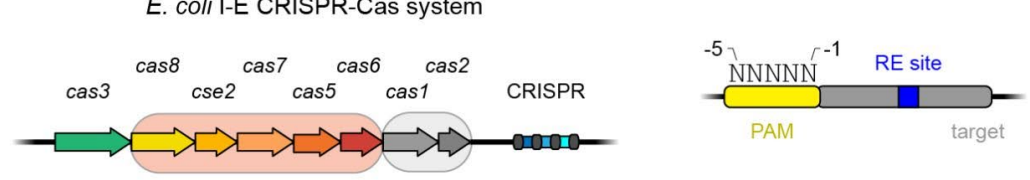

B

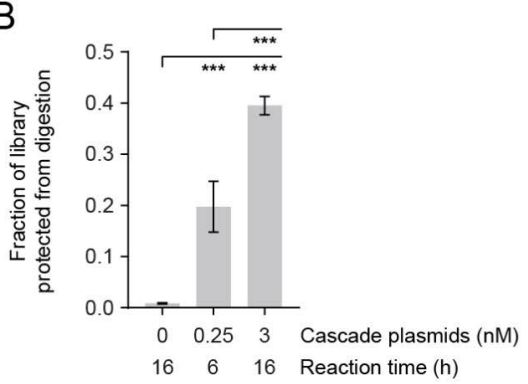

C

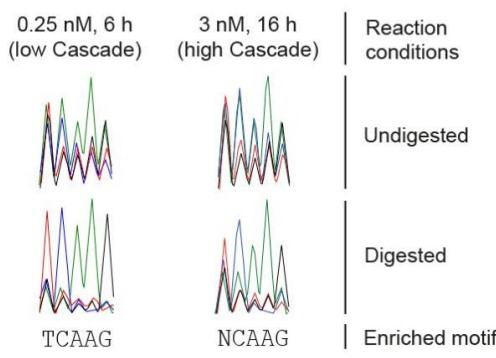

D
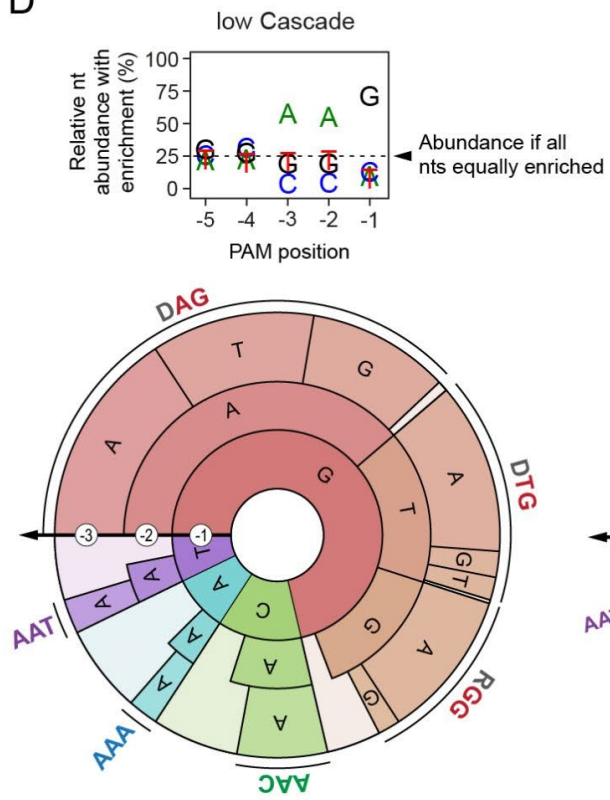
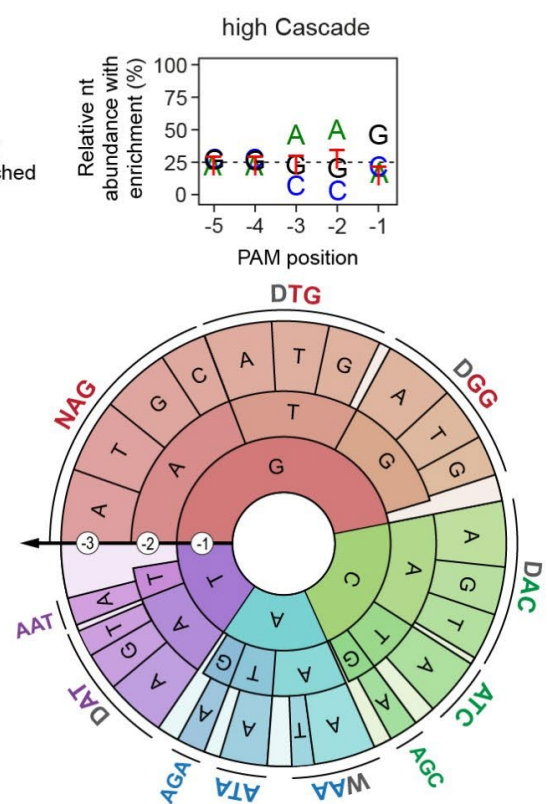

$\mathrm{E}$

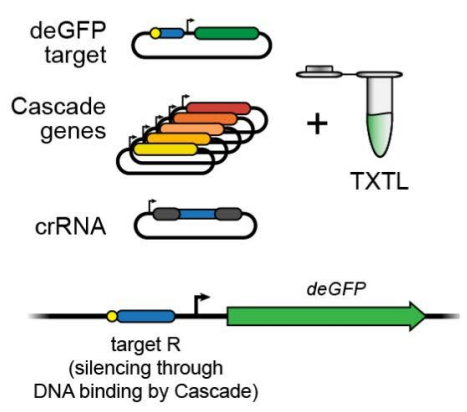

$\mathrm{F}$

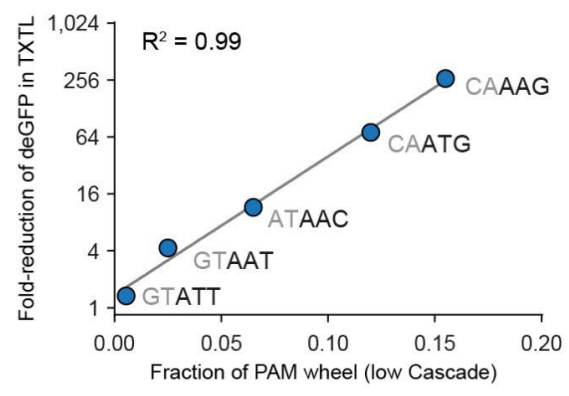

G

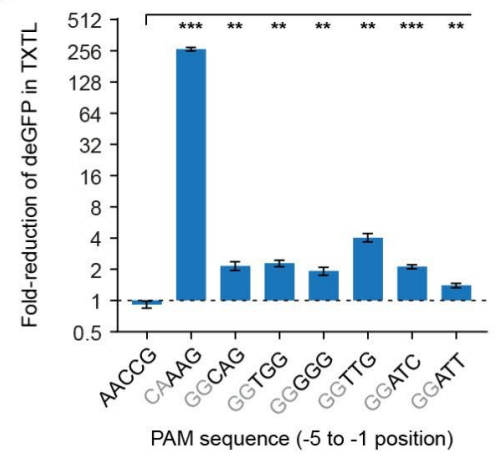

525 Figure 2. Validation of PAM-DETECT with the I-E CRISPR-Cas system from E. coli.

526 (A) The Type I-E CRISPR-Cas systems from E. coli. The genes encoding the Cascade complex

527 are in the light orange box, while the genes encoding the acquisition proteins are in the gray box.

528 Right: $5 \mathrm{~N}$ library of potential PAM sequences used with PAM-DETECT.

529 (B) Extent of PAM library protection under conditions resulting in low or high levels of Cascade

530 based on qPCR. Library protection compares the library with and without RE digestion.

531 (C) Preliminary recognized PAM with low or high levels of Cascade based on Sanger sequencing.

532 Overrepresentation of $\mathrm{T}$ and $\mathrm{C}$ at the -5 and -4 position, respectively, can be explained by the 
533 library generation, as TCAAG represented the most prevalent sequence in the library. As a result,

534 protection of an AAG motive protects the majority of the TCAAG sequences.

535 (D) Nucleotide-enrichment plots and PAM wheels based on conducting PAM-DETECT with low 536 or high levels of Cascade. Individual sequences comprising at least $2 \%$ of the PAM wheel are

537 shown. Results represent the average of duplicate independent experiments. The size of the arc

538 for an individual sequence corresponds to its relative enrichment within the library.

539 (E) Overview of the TXTL-based PAM validation assay. PAM sequences are tested by Cascade

540 binding target $\mathrm{R}$ flanked by the tested PAM. Because target $\mathrm{R}$ overlaps the promoter driving

541 expression of deGFP, target binding would block deGFP expression. Target R is distinct from the

542 restriction site-containing target used with PAM-DETECT.

543 (F) Correlation between PAM enrichment from PAM-DETECT and gene repression in TXTL.

544 Enrichment was based on the fraction of the PAM wheel derived from the low Cascade condition.

545 Enrichment values represent the mean of duplicate PAM-DETECT assays, while fold-reduction

546 values represent the mean of triplicate TXTL assays. Fold-reduction was calculated based on a

547 non-targeting crRNA control.

548 (G) TXTL validation of PAM sequences identified by PAM-DETECT but not by PAM-SCANR.

549 CAAAG serves as a positive control. AACCG matches the 3 ' end of the repeat and therefore 550 serves as a negative control. The AACCG self PAM is the reference for statistical analyses.

551 Error bars in $B$ and $G$ indicate the mean and standard deviation of triplicate independent 552 experiments. ${ }^{* *}: p<0.001{ }^{* *}: p<0.01 .{ }^{*}: p<0.05$. ns: $p>0.05$. 
A S. thermophilus DGCC 7710
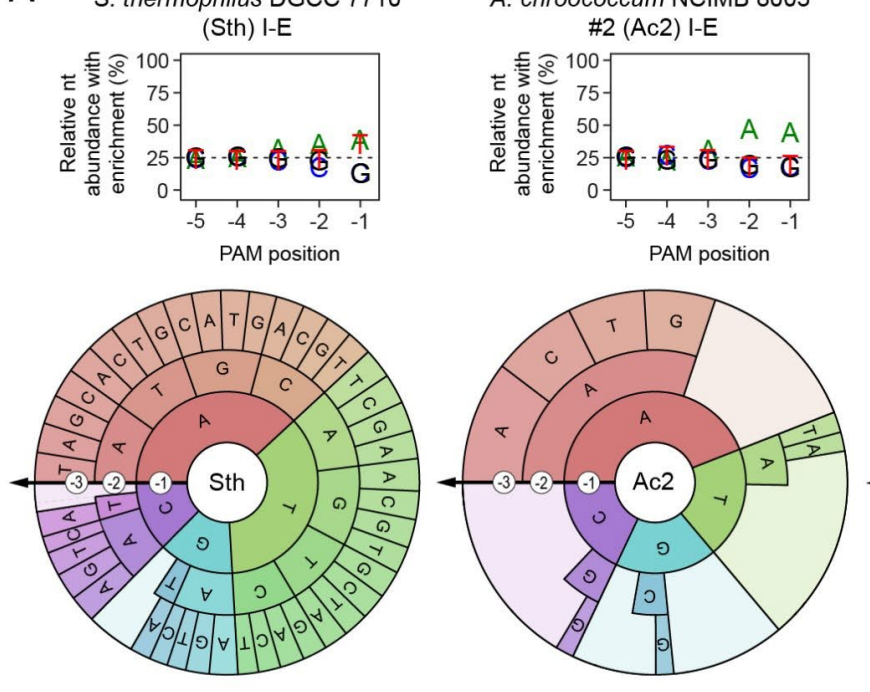

A. chroococcum NCIMB 8003
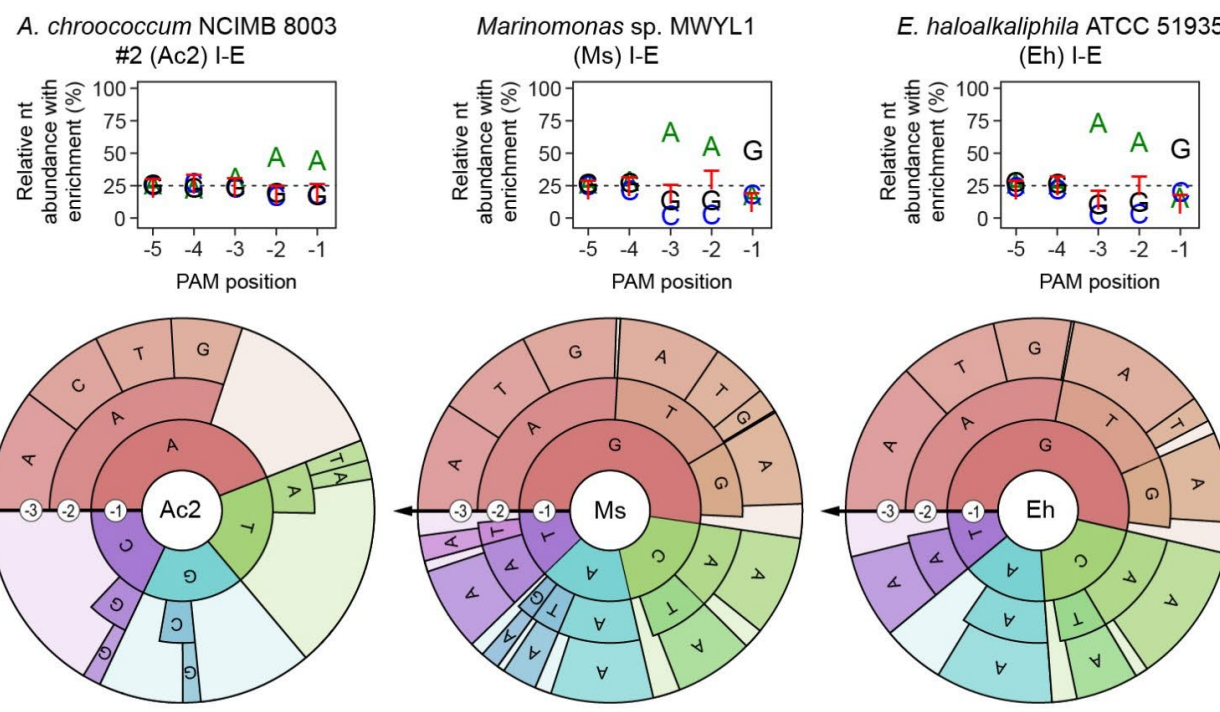

A. chroococcum NCIMB 8003 \#3 (Ac3) I-E
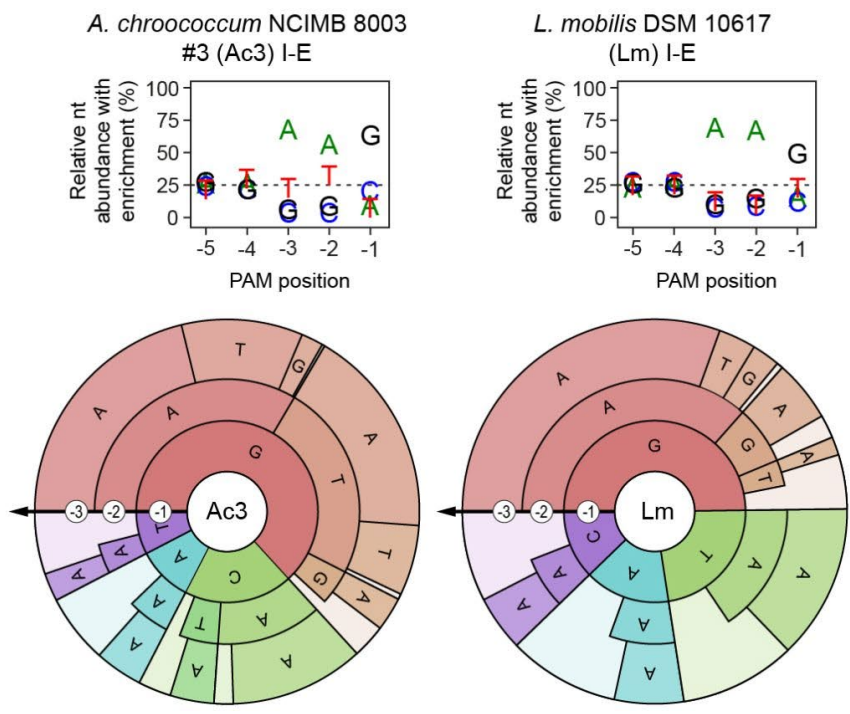

B

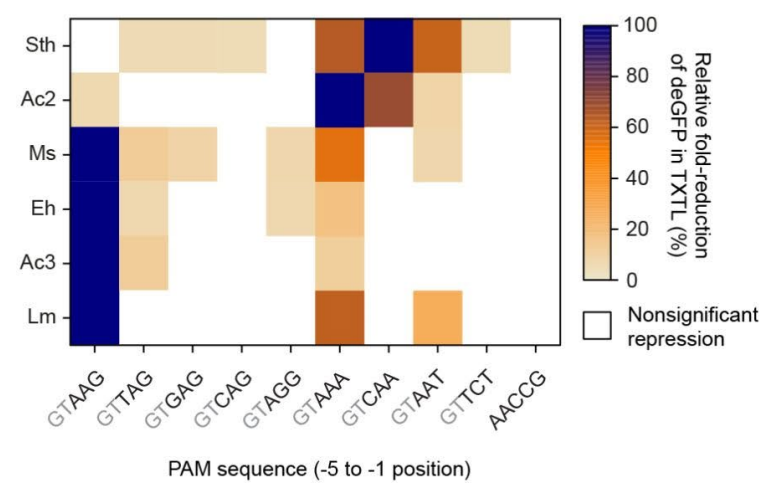

554 Figure 3. Harnessing the functional diversity of I-E CRISPR-Cas systems.

555 (A) Nucleotide enrichment plots and PAM wheels for selected I-E systems subjected to PAM-

556 DETECT. See Figure S1 for 5 additional systems subjected to PAM-DETECT. Ac1 (in Figure S1),

557 Ac2, and Ac3 are present in the same bacterium. Individual sequences comprising at least $2 \%$ of

558 the PAM wheel are shown. Plots and PAM wheels are averages of duplicate independent

559 experiments.

560 (B) Comparison of PAM recognition between systems. Recognition was determined by assessing

561 repression of a deGFP reporter in TXTL. Values represent the mean of three TXTL experiments. 
562 Fold-reduction values that are not significantly different from that of the non-targeting crRNA

563 control $(p>0.05)$ are shown as white squares. The PAM sequence showing the highest fold-

564 reduction for each system was set to $100 \%$. AACCG matches the $3^{\prime}$ end of the repeat for most of

565 the systems. 
bioRxiv preprint doi: https://doi.org/10.1101/2021.10.18.464778; this version posted October 18,2021 . The copyright holder for this preprint (which was not certified by peer review) is the author/funder, who has granted bioRxiv a license to display the preprint in perpetuity. It is made available under aCC-BY-NC-ND 4.0 International license.

A

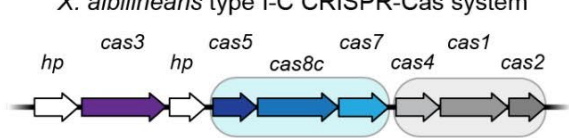

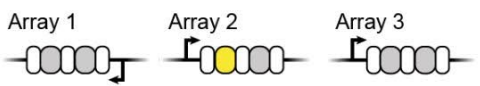

Array 4
Array 5

Array $5 \quad$ Array 6

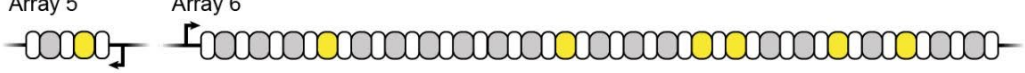

X. albilineans type I-F1 CRISPR-Cas system

cas1

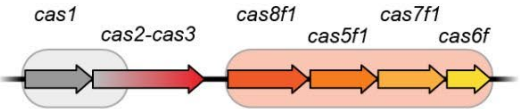

I-C repeat
I-F1 repeat

Self-targeting spacer

Nonself-targeting spacer
B

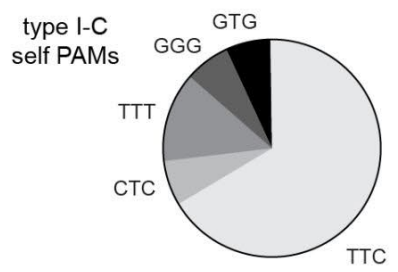

type I-F1 self PAMs

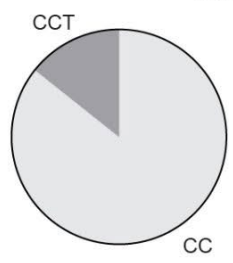

C
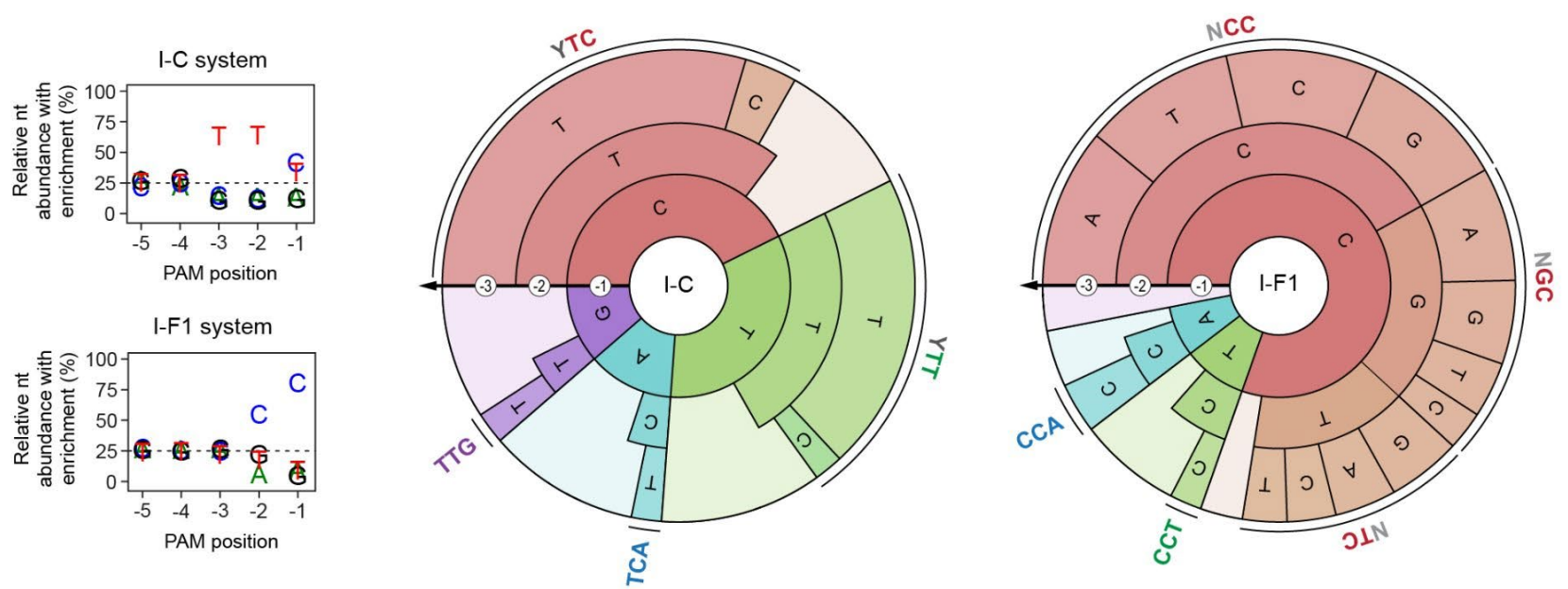

D
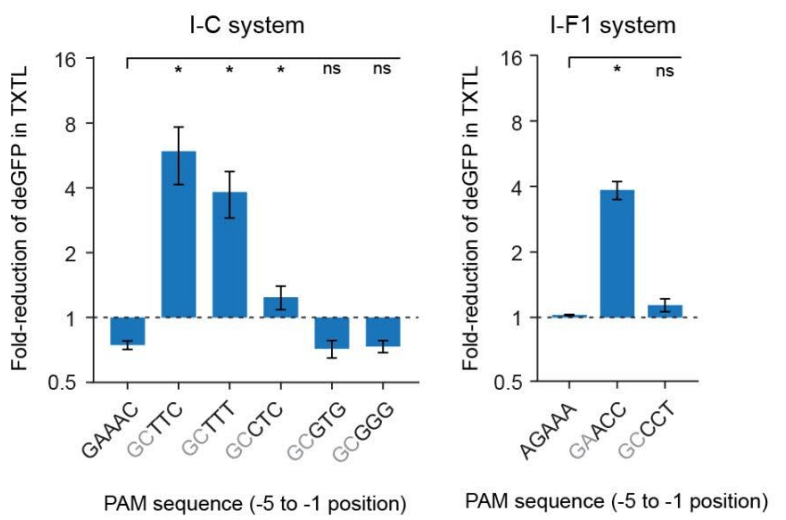

E

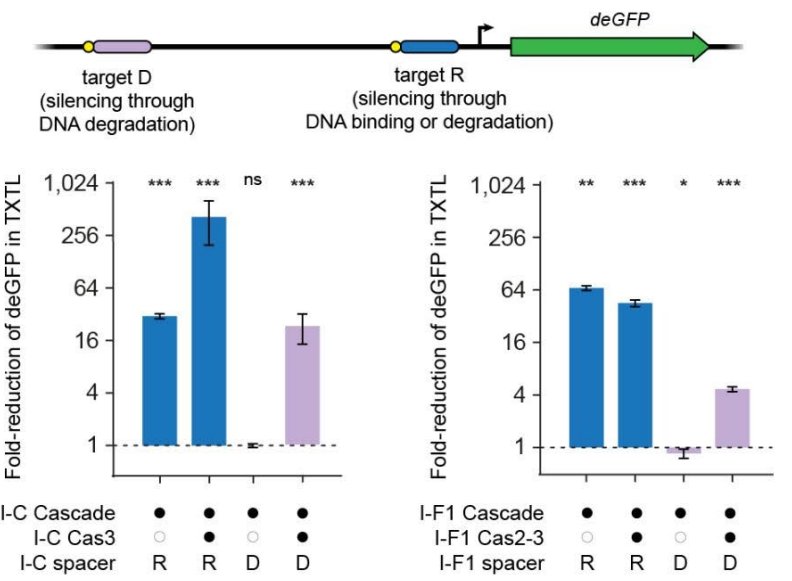

567 Figure 4. Interrogating extensive self-targeting for two type I CRISPR-Cas systems in 568 Xanthomonas albilineans. 
569 (A) Overview of the I-C and I-F1 CRISPR-Cas systems and self-targeting spacers. The genes 570 encoding the Cascade complex are in the light blue box (I-C) or the light orange box (I-F1), while

571 the genes encoding the acquisition proteins are in the gray box.

572 (B) Distribution of PAMs associated with the self-targets. See Figure S2 for the self-target

573 location and Table S4 for the self-target sequences.

574 (C) Nucleotide-enrichment plots and PAM wheels based on conducting PAM-DETECT. Individual 575 sequences comprising at least $2 \%$ of the PAM wheel are shown. Plots and PAM wheels are 576 averages of duplicate independent experiments.

577 (D) Validation of PAMs associated with self-targets in TXTL. Fold-reduction was calculated based 578 on a non-targeting crRNA control. GAAAC and AGAAA match the 3 ' end of the repeat for the I-C 579 and I-F1 systems, respectively. Either self PAM is the reference for statistical analyses.

580 (E) Assessing DNA binding by Cascade and DNA degradation by Cas3 in TXTL. Targeting far 581 upstream of the promoter (target D) can reduce deGFP levels only through degradation of the 582 plasmid. Targeting the promoter (target R) can reduce deGFP levels through DNA binding or 583 plasmid degradation. Fold-reduction was calculated based on a non-targeting crRNA control. The 584 non-targeting crRNA control is the reference for statistical analyses. Target D with only the I-F1 585 Cascade yielded modestly but significantly altered deGFP levels between targeting and non586 targeting conditions, although targeting resulted in an increase in deGFP levels.

587 Errors bars in $D$ and $E$ indicate the mean and standard deviation of triplicate independent 588 experiments. ${ }^{* *}: p<0.001{ }^{* *}: p<0.01 .^{*}: p<0.05$. ns: $p>0.05$. 
A

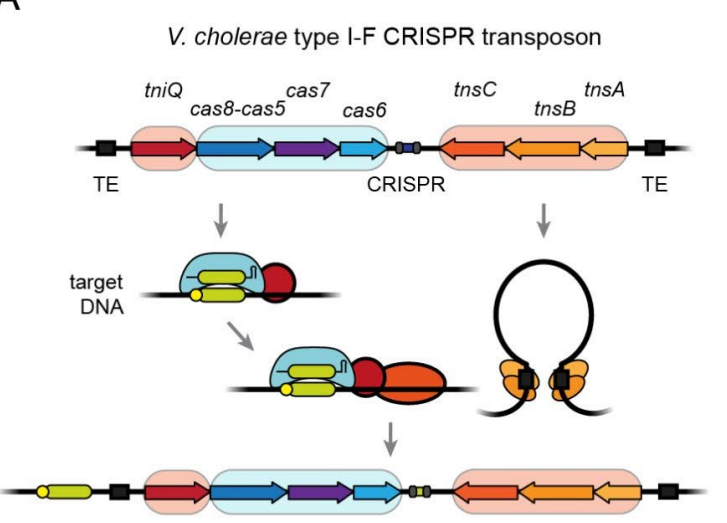

B

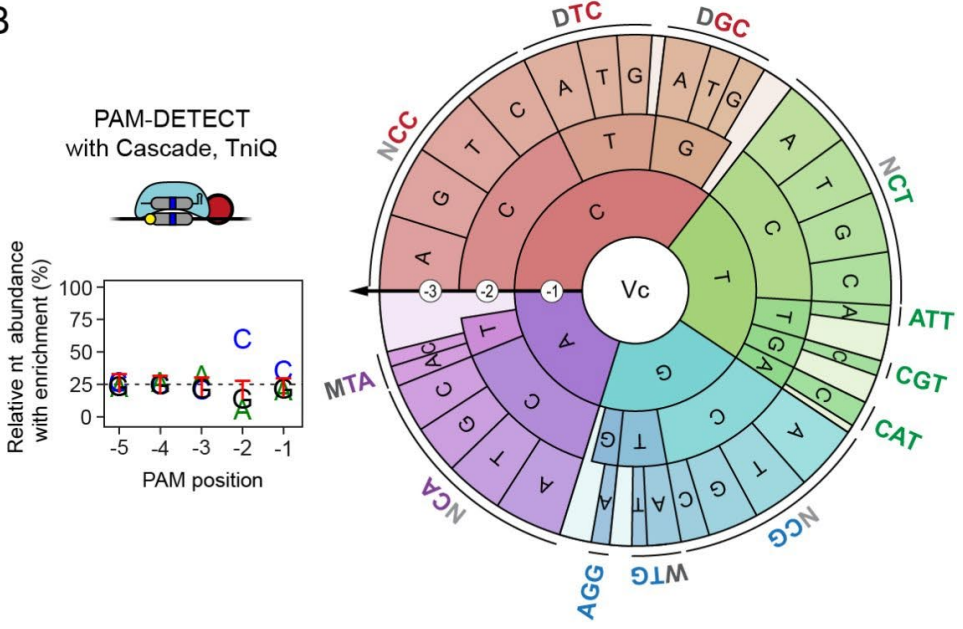

C

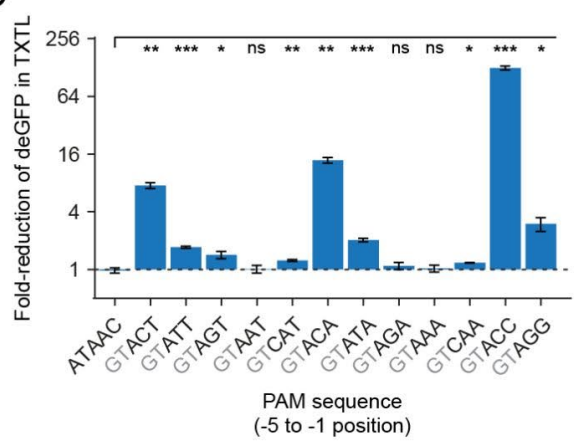

D

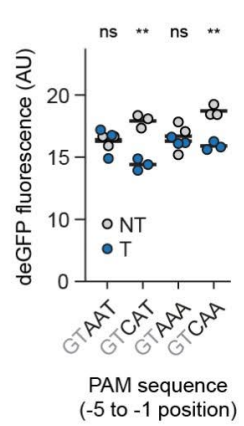

E
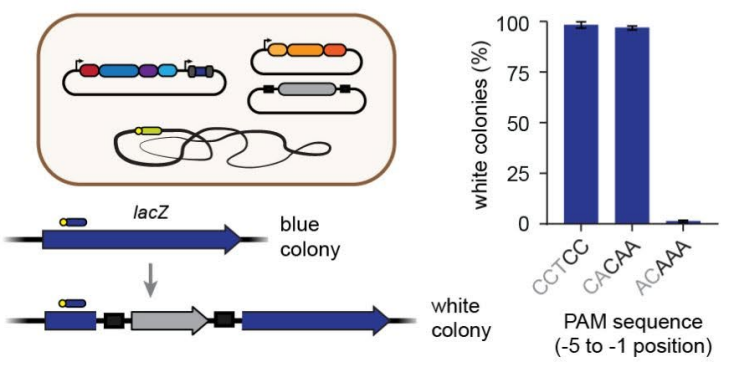

590 Figure 5. Interrogating the PAM profile of the Vibrio cholerae I-F CRISPR transposon.

591 (A) Overview of V. cholerae I-F CRISPR transposon and its mechanism of transposition.

592 (B) Nucleotide-enrichment plot and PAM wheel based on conducting PAM-DETECT with

593 Cascade and TniQ. Individual sequences comprising at least $1 \%$ of the PAM wheel are shown.

594 The plot and PAM wheel are averages of duplicate independent experiments.

595 (C) Validation of PAMs in TXTL. Gene repression was evaluated with Cascade and the indicated

596 PAM flanking target $\mathrm{R}$ upstream of the deGFP reporter. See Figure 2E for details. Fold-reduction

597 was calculated based on a non-targeting crRNA control. ATAAC matches the 3' end of the repeat

598 and therefore serves as a negative control. The ATAAC self PAM is the reference for statistical

599 analyses.

600 (D) Individual measurements of endpoint deGFP levels in TXTL. Triplicate values are shown for

601 selected PAMs with a targeting (T) or non-targeting (NT) crRNA. See C for details. 
602 (E) Validation of PAM recognition for DNA transposition in E. coli. Donor DNA is inserted within

603 the lacZ gene, preventing the formation of blue colonies on IPTG and X-gal. Different targets

604 within lacZ were selected to test the indicated PAM. The targets for the CAA and AAA PAMs are

605 shifted by one nucleotide. See Figure S3 for more information.

606 Error bars in C, D, and E indicate the mean and standard deviation of triplicate independent

607 experiments. ${ }^{* * *}: p<0.001{ }^{* *}: p<0.01{ }^{*}: p<0.05$. ns: $p>0.05$. 
A type I-B2 CRISPR transposons
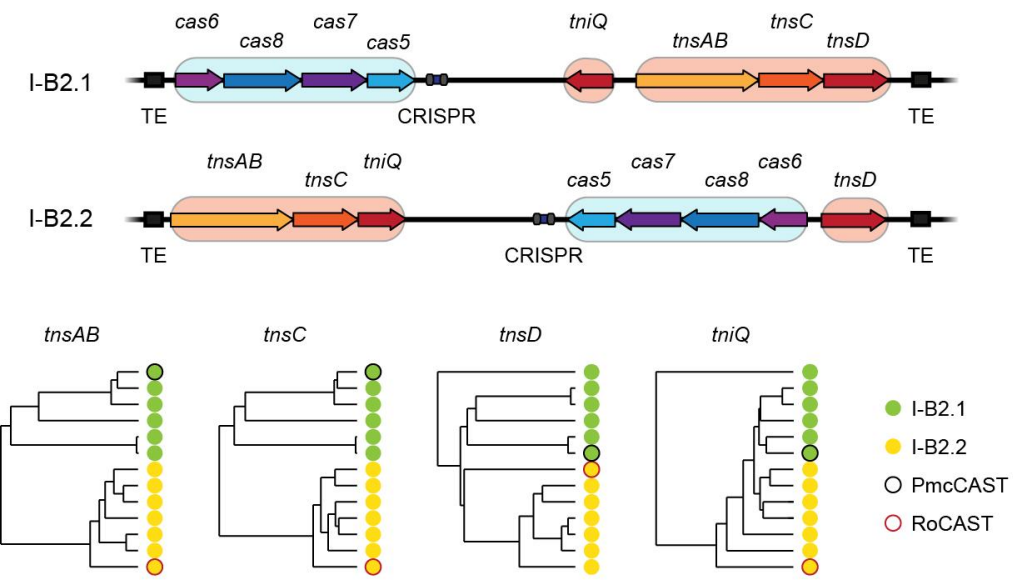

B
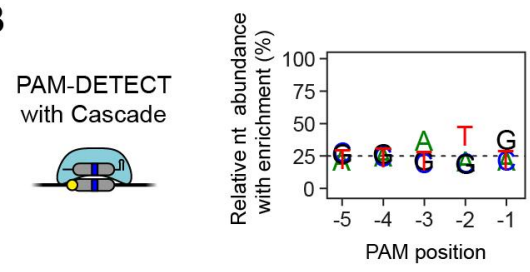

C
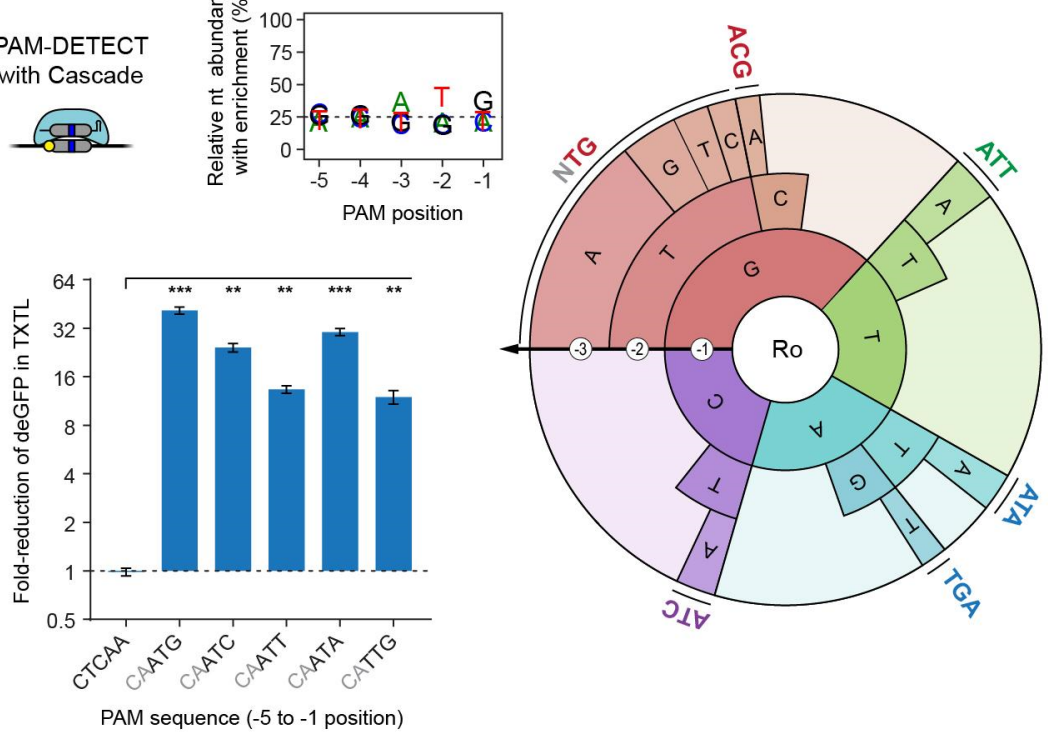

Figure 6: Interrogating PAM requirements of the Rippkaea orientalis I-B2.2 CRISPR transposon.

610 (A) Overview of I-B2.1 and I-B2.2 CRISPR transposons. The two are divided based on the gene

611 organization within each transposon. Phylogenetic trees are shown for the transposon genes. The

612 Peltigera membranacea cyanobiont 210A CRISPR transposon (PmcCAST) from the I-B2.1

613 branch was previously characterized (Saito et al., 2021). The $R$. orientalis CRISPR transposon

614 (RoCAST) from the I-B2.2 branch is characterized in this work. See Supplementary Figure S4 for

615 alignments with names that match the order within the trees. 
616 (B) Nucleotide-enrichment plot and PAM wheel based on conducting PAM-DETECT with

617 Cascade from RoCAST. Individual sequences comprising at least $2 \%$ of the PAM wheel are

618 shown. The plot and PAM wheel are averages of duplicate independent experiments.

619 (C) Validation of PAMs in TXTL. Gene repression was evaluated with Cascade and the indicated

620 PAM flanking target $\mathrm{R}$ upstream of the deGFP reporter. See Figure 2E for details. Fold-reduction

621 was calculated based on a non-targeting crRNA control. CTCAA matches the 3' end of the repeat

622 and therefore serves as a negative control. The CTCAA self PAM is the reference for statistical

623 analyses.

624 Error bars in C indicate the mean and standard deviation of triplicate independent experiments.

$625{ }^{* * *}: p<0.001{ }^{* *}: p<0.01 .^{*}: p<0.05$. ns: $p>0.05$ 
bioRxiv preprint doi: https://doi.org/10.1101/2021.10.18.464778; this version posted October 18,2021 . The copyright holder for this preprint (which was not certified by peer review) is the author/funder, who has granted bioRxiv a license to display the preprint in perpetuity. It is made available under aCC-BY-NC-ND 4.0 International license.

A

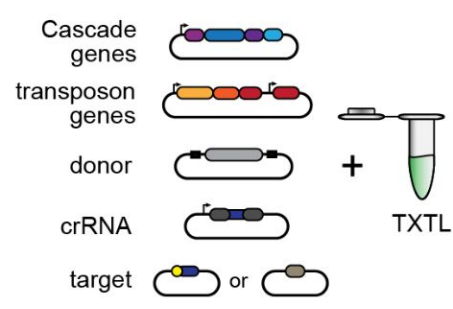

B

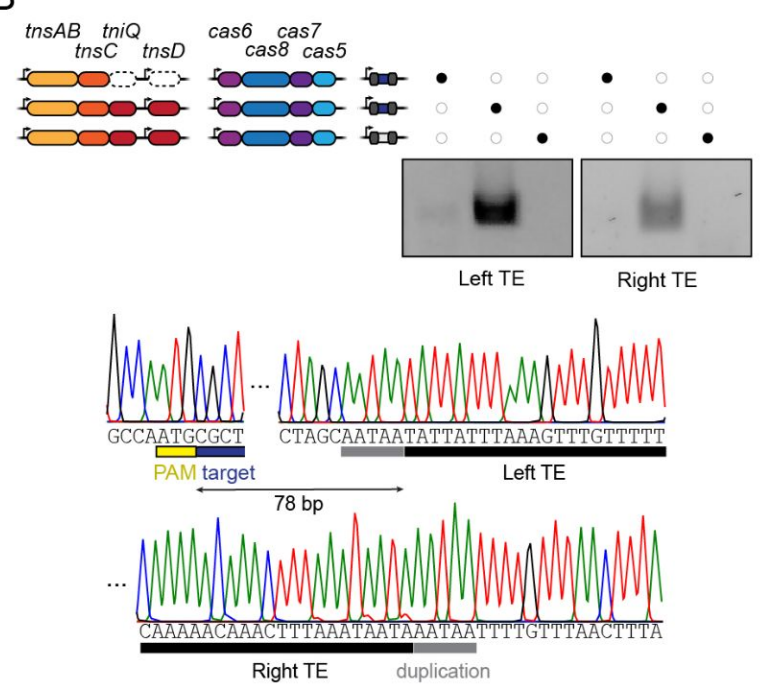

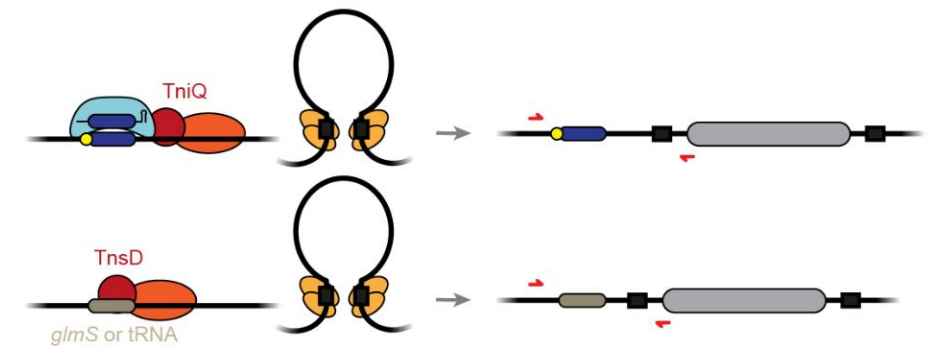

C

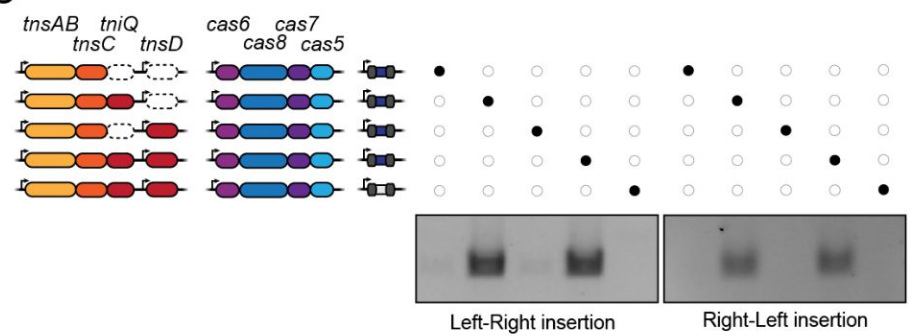

D

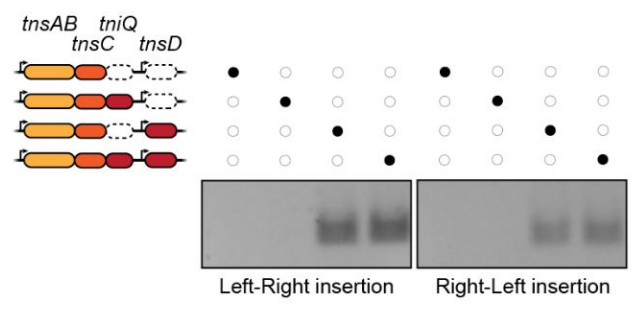

$E$

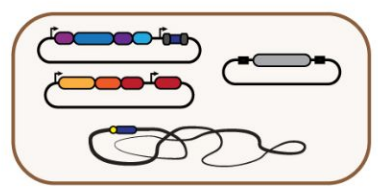

CRISPR-dependent transposition

$\mathrm{F}$

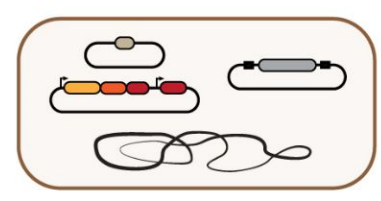

CRISPR-independent transposition

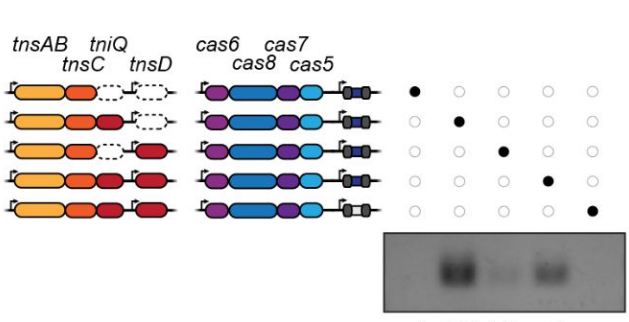

Left-Right insertion

G

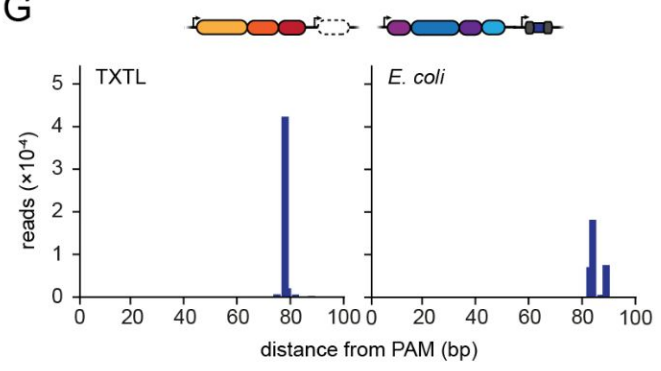

$\mathrm{H}$

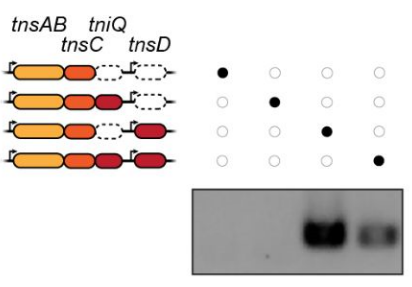

Left-Right insertion

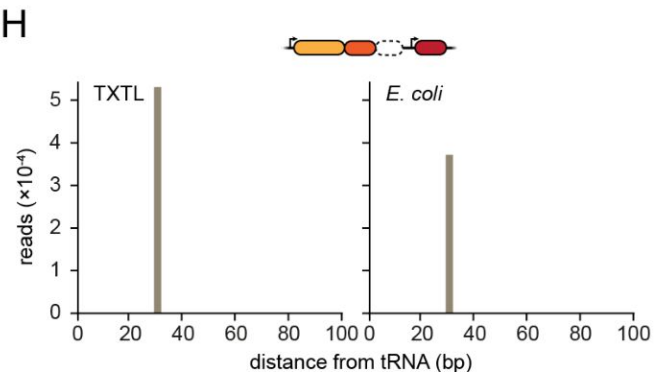

627 Figure 7. Investigating transposition of the Rippkaea orientalis I-B2.2 CRISPR transposon in

TXTL and in E. coli. 
629 (A) Overview of the TXTL-based transposition assay. For I-B CRISPR transposons, transposition

630 can occur through crRNA-guided recognition of a DNA target or through TnsD-guided recognition

631 of $\mathrm{glmS}$ or a tRNA gene independent of the CRISPR machinery. Primers (red) are shown to

632 selectively amplify the transposition product. The $R$. orientalis I-B2.2 CRISPR transposon

633 (RoCAST) flanks the tRNA-Leu gene.

634 (B) CRISPR-dependent transposition and determination of transposon ends and insertion

635 distance using the TXTL-based transposition assay with RoCAST. PCR products are specific to

636 the left-right orientation and span the crRNA target site and the beginning of the cargo (left TE)

637 or the end of the cargo and downstream of the insertion site (right TE).

638 (C) CRISPR-dependent transposition in TXTL. PCR products span the crRNA target site and the

639 beginning of the cargo for both orientations of transposon insertion.

640 (D) CRISPR-independent transposition in TXTL. PCR products span the end of the tRNA-Leu

641 gene and the beginning of the cargo for both orientations of transposon insertion.

642 (E) CRISPR-dependent transposition in E. coli. PCR products span the crRNA target site and the

643 beginning of the cargo (left-right orientation).

644 (F) CRISPR-independent transposition in E. coli. PCR products span the TnsD target site and the

645 beginning of the cargo (left-right orientation).

646 (G) Assessment of insertion distances for CRISPR-dependent transposition in TXTL and in E.

647 coli. The constructs lacking tns $D$ were used. Transposition was determined by next-generation

648 sequencing of the PCR product spanning the crRNA target site and the beginning of the cargo

649 (left-right orientation).

650 (H) Assessment of insertion distances for CRISPR-independent transposition in TXTL and in $E$.

651 coli. The constructs lacking tniQ were used. Transposition was determined by next-generation

652 sequencing of the PCR product spanning the end of the tRNA-Leu gene and the beginning of the

653 cargo (left-right orientation).

654 All gel images are representative of at least duplicate independent experiments. 


\section{STAR METHODS}

\section{METHOD DETAILS}

\section{Plasmid construction}

659 Standard cloning methods Gibson Assembly, Site Directed Mutagenesis (SDM) and Golden Gate 660 were used to clone plasmids used in TXTL experiments. pPAM_library containing a PAM library 661 with five randomized nucleotides was generated by SDM on p70a-deGFP_Pacl with primers 662 FW531 and FW532 (Table S5). Single-spacer CRISPR arrays were generated either with Golden

663 Gate adding spacer sequences in a plasmid containing two repeat sequences interspaced by two 664 Bael or Bbsl restriction sites or by SDM on pEc_gRNA1, pEc_gRNA2 or pEc_gRNAnt to change 665 the repeat sequences to match the tested CRISPR systems. Plasmids harboring different PAM 666 sequences for PAM validation assays were generated by SDM on p70a-deGFP_Pacl. To 667 generate plasmids encoding $X$. albilineans type I-C and type I-F1 Cas proteins, genomic DNA 668 isolated from Xanthomonas albilineans CFBP7063 was PCR amplified using Q5 Hot Start High669 Fidelity 2X Master Mix (NEB) and cloned into pET28a using Gibson Assembly. All other plasmids 670 were generated with Gibson Assembly or SDM (Table S5). All constructed plasmids were verified 671 with Sanger sequencing.

673 For the VcCAST in vivo transposition experiments we cloned into the previously described 674 pSL0284 vector (Klompe et al., 2019) two spacers targeting the lacZ gene of the E. coli BL21 675 (DE3) genome, yielding the pQCas_CAA and pQCas_AAA vectors. The protospacer targeted by 676 the former vector has a 5'CAA PAM, whereas the protospacer targeted by the latter vector has a 677 5'AAA PAM.

678

679 For the RoCAST in vivo transposition experiments, genes encoding the Rippkaea orientalis 680 tns $A B$, tns $C$, tns $D$ and tniQ were synthesized (Twist Bioscience) and cloned in the pET24a vector 
681 in various combinations, resulting in the construction of the pRoTnsABC, pRoTnsABCD,

682 pRoTnsABCQ, pRoTnsABCDQ vectors (Table S5). The Rippkaea orientalis Cascade operon

683 (cas6, cas8, cas7, cas5) was synthesized (Twist Bioscience) and cloned into the pCDFDuet-1

684 vector together with a gfp gene flanked by two Bsal restriction sites and the corresponding

685 CRISPR direct repeats. Into the resulting pRoCascade_gfp vector we cloned a spacer targeting

686 the lacZ gene of the E. coli BL21 (DE3) genome and a non-targeting control spacer, constructing

687 the pRoCascade_T (targeting) and pRoCascade_NT (non-targeting) vectors, respectively (Table

688 S5). DNA fragments encoding the right and left RoCAST ends were synthesized (IDT) and cloned

689 into the pUC19 vector flanking a gfp gene, yielding pRoDonor (Table S5). A 105-bp long DNA

690 fragment from the Rippkaea orientalis genome, encoding the region which is located right

691 upstream of the left end of RoCAST and includes the last 74 bp of the tRNA-Leu gene, was

692 synthesized (IDT) and cloned into the pCDFDuet-1 vector, resulting in the construction of the

693 pRoTarget vector (Table S5).

694

695 PAM-DETECT

696 A plasmid with five randomized nucleotides flanking a target site covering a Pacl restriction

697 enzyme recognition site was constructed as described before. If Cas proteins required for

698 Cascade formation were encoded on separate plasmids, a MasterMix with the required Cas

699 protein encoding plasmids in their stoichiometric amount was prepared beforehand. Thereby, a

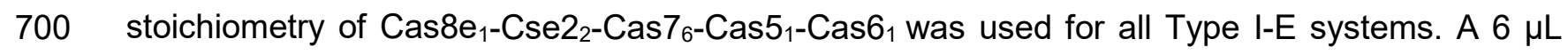

701 TXTL reaction was assembled consisting of $3 \mathrm{nM}$ (high Cascade) or $0.25 \mathrm{nM}$ (low Cascade) of

702 the Cascade-encoding plasmid or the Cascade MasterMix, 4.5 $\mu \mathrm{L}$ myTXTL Sigma 70 Master Mix,

$703 \quad 0.2$ nM pET28a_T7RNAP, 0.5 mM IPTG, 1 nM gRNA-encoding plasmid and 1 nM pPAM_library.

704 A negative control containing all components from the reaction besides the Cascade plasmids

705 and the gRNA-expressing plasmid was included. PAM-DETECT assays assessing either the type

706 I-C or the type I-F1 system in X. albilineans were lacking IPTG in their reactions. TXTL reactions 
707 were incubated at $29^{\circ} \mathrm{C}$ for $6 \mathrm{~h}$ or $16 \mathrm{~h}$. The samples were diluted 1:400 in nuclease-free $\mathrm{H} 2 \mathrm{O}$.

$708500 \mu \mathrm{L}$ were digested at $37^{\circ} \mathrm{C}$ with Pacl (NEB) at 0.09 units $/ \mu \mathrm{L}$ in $1 \times$ CutSmart Buffer (NEB) for 1

$709 \mathrm{~h}$ and $500 \mu \mathrm{L}$ were used as a "non-digested" control by adding nuclease-free $\mathrm{H} 2 \mathrm{O}$ instead of Pacl.

710 After inactivation of Pacl at $65^{\circ} \mathrm{C}$ for $20 \mathrm{~min}, 0.05 \mathrm{mg} / \mathrm{mL}$ Proteinase $\mathrm{K}$ (GE Healthcare) was added

711 and incubated at $45^{\circ} \mathrm{C}$ for $1 \mathrm{~h}$. After inactivation of Proteinase $\mathrm{K}$ at $95^{\circ} \mathrm{C}$ for 5 min, remaining

712 plasmids were extracted via standard EtOH precipitation. Illumina adapters with unique dual

713 indices were added by two amplification steps with KAPA HiFi HotStart Library Amplification Kit

714 (KAPA Biosystems) and purified by Agencourt AMPure XP (Beckman Coulter) after every PCR

715 reaction. The first PCR reaction adds the Illumina sequencing primers with primers that can be

716 found in Table S5 using $15 \mu \mathrm{L}$ of the EtOH-purified samples in a $50 \mu \mathrm{L}$ reaction and 19 cycles.

717 The second PCR adds the unique dual indices and the flow cell binding sequence using $1 \mathrm{ng}$

718 purified amplicons generated with the first PCR using 18 cycles. The samples were submitted for

719 next-generation sequencing with 50 bp paired-end reads with 1.25 or 2.0 million reads per sample

720 on an Illumina NovaSeq 6000 sequencer. PAM wheels were generated according to Leenay et

721 al. (Leenay et al., 2016). Nucleotide enrichment plot generation was adapted to the script from

722 Marshall et al. (Marshall et al., 2018) by changing the script to visualize the probability of a given

723 nucleotide at a given position by depicting the percentage of the nucleotide in that position. All

724 PAM-DETECT assays were done in duplicates and PAM wheel and nucleotide enrichment plots

725 show averages. The generated NGS data have been deposited in NCBI's Gene Expression

726 Omnibus (Edgar et al., 2002) and are accessible through GEO Series accession number

727 GSE179614 (https://www.ncbi.nlm.nih.gov/geo/query/acc.cgi?acc=GSE179614 ). The following

728 token can be used to access the data prior to publication: exexiqgyhrcblgj.

729

730 qPCR Reactions

731 To assess the remaining amount of PAM-library containing plasmid after conducting PAM-

732 DETECT, quantitative PCR (qPCR) was performed using SsoAdvanced Universal SYBR Green 
733 Supermix (Biorad) in $10 \mu \mathrm{L}$ reactions. The reactions were quantified using a QuantStudio Real-

734 Time PCR System (Thermo Fisher) with an annealing temperature of $68{ }^{\circ} \mathrm{C}$ according to

735 manufacturers' instructions. All samples were prepared by using the liquid handling machine

736 Echo525 (Beckman Coulter).

737

738

deGFP repression assays in TXTL

739 To assess activity of CRISPR-Cas systems, deGFP-repression assays in $3 \mu \mathrm{L}$ or $5 \mu \mathrm{L}$ TXTL

740 reactions were conducted, measuring deGFP-expression over time in a 96-well V-bottom plate

741 with BioTek Synergy H1 plate reader (BioTek) at 485/528 nm excitation/emission (Shin and

742 Noireaux, 2012). All TXTL samples were either prepared by hand or by using the liquid handling

743 machine Echo525 (Beckman Coulter).

$7453 \mu \mathrm{L}$ TXTL reactions for PAM validation assays were prepared containing Cascade plasmid 746 concentrations according to Table S2. If Cas proteins required for Cascade formation were 747 encoded on separate plasmids, a MasterMix with the required Cas protein encoding plasmids in 748 their stoichiometric amount was prepared beforehand. Thereby, a stoichiometry of Cas $5{ }_{1}-$ Cas $_{1} 1^{-}$ $749 \mathrm{Cas}_{7}$ was used for $X$. albilineans Type I-C, Cas $8 \mathrm{f}_{1}$-Cas $5 \mathrm{f} 1_{1}-$ Cas $7 \mathrm{f} 1_{6}-\mathrm{Cas}_{6} \mathrm{f}_{1}$ was used for $X$. 750 albilineans Type I-F1 and Cas8e ${ }_{1}-\mathrm{Cse} 2_{2}-$ Cas7 $_{6}$-Cas5 ${ }_{1}-\mathrm{Cas}_{1}$ was used for all Type I-E systems.

751 Other components included in the TXTL reactions were $2.25 \mu \mathrm{L}$ myTXTL Sigma 70 Master Mix, $7520.2 \mathrm{nM}$ p70a_T7RNAP, $0.5 \mathrm{mM}$ IPTG and $1 \mathrm{nM}$ gRNA-encoding plasmid. After a $4 \mathrm{~h}$ pre753 incubation at $29^{\circ} \mathrm{C}$ or $37^{\circ} \mathrm{C}$ that allowed the ribonucleoprotein complex of Cascade and crRNA

754 to form, $1 \mathrm{nM}$ reporter plasmid (pGFP_XXXXX) with various PAM sequences in close proximity 755 to the promoter driving deGFP expression was added to the reaction to ensure Cascade-binding 756 would lead to deGFP inhibition. The reactions were incubated for additional $16 \mathrm{~h}$ at $29^{\circ} \mathrm{C}$ or 37 $757{ }^{\circ} \mathrm{C}$ while measuring deGFP expression. The gRNAs were constructed to target a protospacer 758 within the degfp promoter located adjacent to the various PAM sequences. 
760 To test the cleavage and/or binding ability of the type I-C and the type I-F1 systems in $X$.

761 albilineans, $3 \mu \mathrm{L}$ TXTL assays were conducted containing Cascade-encoding plasmids in the

762 stoichiometry as mentioned before. To test binding ability, 2.25 $\mu \mathrm{L}$ myTXTL Sigma 70 Master Mix,

7630.2 nM p70a_T7RNAP, 0.5 mM IPTG, 1 nM gRNA1-, gRNA2, or gRNAnt-encoding plasmid and

$7641 \mathrm{nM}$ or $0.25 \mathrm{nM}$ Cascade MasterMix was added to a TXTL reaction for the type I-C and type I-

765 F1 system, respectively. To test cleavage ability, $2.25 \mu \mathrm{L}$ myTXTL Sigma 70 Master Mix, $0.2 \mathrm{nM}$

766 p70a_T7RNAP, 0.5 mM IPTG, 1 nM gRNA1-, gRNA2, or gRNAnt-encoding plasmid, 1 nM

767 Cascade MasterMix and $0.5 \mathrm{nM}$ or $0.25 \mathrm{nM}$ pXalb_IC_Cas3 or pXalb_IF_Cas2-3 was added to a

768 TXTL reaction for the type I-C and type I-F1 system, respectively. After $4 \mathrm{~h}$ pre-expression at

$76929^{\circ} \mathrm{C}, 1 \mathrm{nM}$ p70a_deGFP reporter plasmid was added to the reactions and incubated for

770 additional $16 \mathrm{~h}$ at $29^{\circ} \mathrm{C}$ while measuring deGFP-fluorescence. gRNA1 is designed to target a

771 protospacer within the promoter driving deGFP expression adjacent to a type I-C TTC or a type

772 I-F1 CC PAM to ensure Cascade-binding would lead to deGFP-inhibition. gRNA2 is designed to

773 target a protospacer adjacent to a type I-C TTC or a type I-F1 CC PAM upstream of the promoter

774 to ensure cleavage of the targeted plasmid would result in deGFP-inhibition whereas binding-only

775 would result in deGFP-production. gRNAnt represents a non-targeting control.

$7775 \mu \mathrm{L}$ TXTL reactions assessing dispensability of TniQ for $V$. cholerae I-F CAST Cascade-binding

778 were performed with reactions containing $3.75 \mu \mathrm{L}$ myTXTL Sigma 70 Master Mix, $0.2 \mathrm{nM}$

779 p70a_T7RNAP, $0.5 \mathrm{mM}$ IPTG and $0.5 \mathrm{nM}$ pVch_IF_CasQ_gRNA3/nt or 0.5 nM

780 pVch_IF_Cas_gRNA3/nt. After a $4 \mathrm{~h}$ pre-incubation step at $29{ }^{\circ} \mathrm{C}$, the reporter plasmid

781 p70a_deGFP was added and the reactions were incubated for additional $16 \mathrm{~h}$ at $29{ }^{\circ} \mathrm{C}$ while

782 measuring deGFP-fluorescence. gRNA3 is designed to target a protospacer within the promoter

783 driving deGFP expression adjacent to a CC PAM. gRNAnt represents a non-targeting control. 


\section{Transposition in TXTL}

786 To assess crRNA-dependent transposition of the Vibrio cholerae Tn6677 I-F CAST in TXTL, $5 \mu \mathrm{L}$

787 TXTL reactions containing $3.75 \mu \mathrm{L}$ myTXTL Sigma 70 Master Mix, $0.2 \mathrm{nM}$ p70a_T7RNAP, 0.5

$788 \mathrm{mM}$ IPTG, $1 \mathrm{nM}$ of the previously described donor plasmid (pSL0527), $2 \mathrm{nM}$ of the previously

789 described TnsABC-plasmid (pSL0283) (Klompe et al., 2019), 1 nM p70a_deGFP and 1 nM

790 pVch_IF_CasQ_gRNA3 or pVch_IF_CasQ_gRNAnt were prepared. The reactions were

791 incubated at $29^{\circ} \mathrm{C}$ for $16 \mathrm{~h}$. Transposition events were detected in a 1:400 dilution of the TXTL

792 reaction by PCR amplification using Q5 Hot Start High-Fidelity 2X Master Mix (NEB) and

793 combinations of donor DNA and genome specific primers. Transposition was verified by Sanger

794 sequencing (Table S5).

795

796 crRNA-dependent transposition of RoCAST in TXTL was performed in $3 \mu \mathrm{L}$ TXTL reactions 797 consisting of $2.25 \mu \mathrm{L}$ myTXTL Sigma 70 Master Mix, $0.2 \mathrm{nM}$ p70a_T7RNAP, $0.5 \mathrm{mM}$ IPTG, $1 \mathrm{nM}$ 798 pRoCascade, 1 nM pRo_gRNA2/nt, 1 nM pGFP_CAATG, 1 nM pRoDonor or 799 pRoDonor_extended and $1 \mathrm{nM}$ pRoTnsABC, pRoTnsABCD, pRoTnsABCQ or pRoTnsABCDQ.

800 The reactions were incubated at $29^{\circ} \mathrm{C}$ for $16 \mathrm{~h}$. Transposition events were detected in a 1:100

801 dilution of the TXTL reaction by PCR amplification using Q5 Hot Start High-Fidelity 2X Master Mix 802 (NEB) and combinations of donor DNA and genome specific primers (Table S5). Transposition 803 was verified by Sanger sequencing.

805 crRNA-independent transposition of RoCAST in TXTL was performed in $3 \mu \mathrm{L}$ TXTL reactions 806 consisting of $2.25 \mu \mathrm{L}$ myTXTL Sigma 70 Master Mix, 0.2 nM p70a_T7RNAP, 0.5 mM IPTG, 1 nM 807 pRoTarget, $1 \mathrm{nM}$ pRoDonor and $1 \mathrm{nM}$ pRoTnsABC, pRoTnsABCD, pRoTnsABCQ or $808 \mathrm{pRoTns} A B C D Q$. The reactions were incubated at $29{ }^{\circ} \mathrm{C}$ for $16 \mathrm{~h}$. Transposition events were 809 detected in a 1:100 dilution of the TXTL reaction by PCR amplification using Q5 Hot Start High- 
810 Fidelity 2X Master Mix (NEB) and combinations of donor DNA and genome specific primers

811 (Table S5). Transposition was verified by Sanger sequencing.

813 Transposition in vivo

814 For the crRNA-dependent transposition in vivo using the I-F CAST from Vibrio cholerae Tn6677,

815 we employed the previously described transposition system (Klompe et al., 2019). We

816 electroporated $30 \mathrm{ng}$ of the pSL0283 vector with $30 \mathrm{ng}$ of the pSL0527 vector and $30 \mathrm{ng}$ of either

817 the pQCas_CAA or pQCas_AAA vector into E. coli BL21(DE3) electrocompetent cells. We plated

818 a fraction of each electroporation mixture on $100 \mathrm{mg} / \mathrm{ml}$ ampicillin, $50 \mathrm{mg} / \mathrm{ml}$ spectinomycin, 50

$819 \mathrm{mg} / \mathrm{ml}$ kanamycin, $0.1 \mathrm{mM}$ IPTG and $100 \mu \mathrm{g} / \mathrm{ml} \mathrm{X-gal} \mathrm{containing} \mathrm{LB-agar} \mathrm{plates.} \mathrm{The} \mathrm{plates} \mathrm{were}$

820 incubated for $24 \mathrm{~h}$ at $30^{\circ} \mathrm{C}$ and the formed colonies were subjected to blue/white screening.

821 Transposition events were identified by colony PCR using Q5 Hot Start High-Fidelity 2X Master

822 Mix (NEB) and genome specific primers (Table S5).

823

824 For the crRNA-dependent transposition in vivo using RoCAST, we electroporated 30 ng of either 825 pRoCascade_T or pRoCascade_NT vector with $30 \mathrm{ng}$ of pRoDonor and $30 \mathrm{ng}$ of either 826 pRoTnsABC, pRoTnsABCD, pRoTnsABCQ or pRoTnsABCDQ vector into E. coli BL21(DE3)

827 electrocompetent cells. We plated a fraction of each electroporation mixture on $100 \mathrm{mg} / \mathrm{ml}$ 828 ampicillin, $50 \mathrm{mg} / \mathrm{ml}$ spectinomycin, and $50 \mathrm{mg} / \mathrm{ml}$ kanamycin containing LB-agar plates. The 829 plates were incubated for $20 \mathrm{~h}$ at $37^{\circ} \mathrm{C}$ and the formed colonies were scraped and resuspended 830 in LB liquid medium. A fraction of each cell suspension was re-plated on LB-agar plates 831 supplemented with $100 \mathrm{mg} / \mathrm{ml}$ ampicillin, $50 \mathrm{mg} / \mathrm{ml}$ spectinomycin, $50 \mathrm{mg} / \mathrm{ml}$ kanamycin and 0.01 832 mM IPTG for induction of the expression of the Cascade and transposase proteins. The plates 833 were incubated $20 \mathrm{~h}$ at $37^{\circ} \mathrm{C}$ and all the formed colonies were scraped and resuspended in LB 834 liquid medium. A fraction of each cell suspension was subjected to gDNA isolation using the 835 illustra Bacteria genomicPrep Mini Spin Kit (GE Healthcare). Transposition events were identified 
by PCR using Q5 Hot Start High-Fidelity 2X Master Mix (NEB) and combinations of donor DNA

837 and genome specific primers (Table S5).

839 For the crRNA-independent in vivo transposition using RoCAST, we electroporated 30 ng of the

840 pRoTarget with $30 \mathrm{ng}$ of pRoDonor and $30 \mathrm{ng}$ of either the pRoTnsABC, pRoTnsABCD,

$841 \mathrm{pRoTns} A B C Q$ or pRoTnsABCDQ vector into E. coli BL21(DE3) electrocompetent cells. We plated

842 a fraction of each electroporation mixture on $100 \mathrm{mg} / \mathrm{ml}$ ampicillin, $50 \mathrm{mg} / \mathrm{ml}$ spectinomycin, and

$84350 \mathrm{mg} / \mathrm{ml}$ kanamycin containing LB-agar plates. The plates were incubated for $20 \mathrm{~h}$ at $37^{\circ} \mathrm{C}$ and

844 the formed colonies were scraped and resuspended in LB liquid medium. A fraction of each cell

845 suspension was re-plated on LB-agar plates supplemented with $100 \mathrm{mg} / \mathrm{ml}$ ampicillin, $50 \mathrm{mg} / \mathrm{ml}$

846 spectinomycin, $50 \mathrm{mg} / \mathrm{ml}$ kanamycin and $0.01 \mathrm{mM}$ IPTG for induction of the expression of the

847 transposase proteins. The plates were incubated $20 \mathrm{~h}$ at $37^{\circ} \mathrm{C}$ and all the formed colonies were

848 scraped and resuspended in LB liquid medium. A fraction of each cell suspension was subjected

849 to gDNA isolation using the illustra Bacteria genomicPrep Mini Spin Kit (GE Healthcare).

850 Transposition events were identified by PCR using Q5 Hot Start High-Fidelity 2X Master Mix

851 (NEB) and combinations of donor DNA and pRoTarget specific primers (Table S5).

\section{Assessing transposition insertion point}

854 To assess the exact insertion point of Rippkaea orientalis I-B2.2 CAST, in vivo and in vitro,

855 transposition assays were conducted as previously described and the transposition products were

856 PCR amplified and sent for next-generation sequencing. Illumina adapters with unique dual

857 indices were added by two amplification steps with KAPA HiFi HotStart Library Amplification Kit

858 (KAPA Biosystems) and each amplicon was purified by Agencourt AMPure XP (Beckman

859 Coulter). The first PCR reaction adds the Illumina sequencing primer sites with primers that can

860 be found in Table S5, the second PCR adds the unique dual indices and the flow cell binding

861 sequences. $2 \mu \mathrm{L}$ of 1:100 dilutions were used in a $50 \mu \mathrm{L}$ PCR reaction to amplify TXTL reactions 
using either 19 or 30 cycles. 50 ng of genomic DNA were used in a $50 \mu \mathrm{L}$ PCR reaction to amplify

863 in vivo transposition with either 19 or 30 cycles. $1 \mathrm{ng}$ of purified TXTL or in vivo-amplicon were

864 subjected to the second PCR using 18 cycles. Library-pools consisting of six samples were

865 submitted for next-generation sequencing with 300 paired-end reads with 0.15 million reads on

866 an Illumina MiSeq machine.

868 The generated NGS data have been deposited in NCBI's Gene Expression Omnibus (Edgar et 869 al., 2002) and are accessible through GEO Series accession number GSE179614

870 (https://www.ncbi.nlm.nih.gov/geo/query/acc.cgi?acc=GSE179614). The following token can be

871 used to access the data prior to publication: exexiqgyhrcblgj.

QUANTIFICATION AND STATISTICAL ANALYSIS

\section{4 deGFP repression assays in TXTL}

875 The fluorescence background was subtracted from the endpoint deGFP values with TXTL samples consisting of only myTXTL Sigma 70 Master Mix and nuclease-free water. The resulting

877 endpoint deGFP values were either depicted as averages of a targeting gRNA and a non-targeting

878 gRNA or fold change-repression was calculated by the ratio of non-targeting over the targeting 879 deGFP values. Significance was calculated with Welch's t-test. $P>0.05$ is shown as ns, $P<0.05$ 880 is shown as * $P<0.01$ is shown as ${ }^{* *}$ and $P<0.001$ is shown as ${ }^{* * *}$. Within the PAM validation

881 assays represented as fold changes, significance was calculated between the fold change of a 882 given PAM and the fold change of a PAM that corresponds to the 3 ' end of the repeat of the tested 883 CRISPR system. The fold changes of the PAM validation in Fig. 3B are depicted in a heat map.

884 Thereby a difference between a non-targeting sample and a targeting sample with a specific PAM 885 resulting in $\mathrm{P}>0.05$ is shown in white and excluded from further analysis. For all other samples 886 within the heat map, the fold changes were calculated as mentioned above and presented relative 887 to the highest fold change within one system. Significance within the deGFP repression assays 
888 testing binding and cleavage ability of the type I-C and the type I-F1 system in X. albilineans was

889 calculated with the targeting and non-targeting sample for each condition. For the endpoint

890 measurements in Fig. 5C, significance was calculated between a non-targeting sample and a

891 targeting sample targeting the same PAM.

892

893 qPCR

894 Cq values were used to measure target amounts. To calculate the relative abundance of the PAM

895 library containing plasmid in the digested sample to the non-digested sample, the relative plasmid

896 amount was normalized to a control amplifying the pET28a-T7RNAP that has no Pacl recognition

897 site using the the $2^{\wedge}(-(\mathrm{ddCt})$ method. Significance to the control sample lacking a CRISPR-Cas

898 system was calculated with Welch's t-test. $\mathrm{P}>0.05$ is shown as ns, $\mathrm{P}<0.05$ is shown as *, $\mathrm{P}<$

8990.01 is shown as ** and $\mathrm{P}<0.001$ is shown as ***.

900

901 Assessing transposition insertion point

$902 \sim 15$ nts long sequences 5' of the transposon terminal left end were extracted, counted and sorted.

903 The sequences were mapped to the targeted plasmid or the targeted genome tolerating 2 nts

904 mismatches and the distance between the insertion point and the PAM upstream of the

905 protospacer or the end of the $t R N A-L e u$ gene was noted. To only depict reliable insertion points,

906 we present insertion points with more than 20 reads. The insertion points are shown as bar

907 graphs.

908

909 The processed NGS data have been deposited in NCBI's Gene Expression Omnibus (Edgar et

910 al., 2002) and are accessible through GEO Series accession number GSE179614

911 (https://www.ncbi.nlm.nih.gov/geo/query/acc.cgi?acc=GSE179614). The following token can be

912 used to access the data prior to publication: exexiqgyhrcblgj.

913 


\section{In silico selection of representative type I-E CRISPR-Cas systems for PAM-DETECT}

915 HMM profiles for the Cas5e, Cas6e, Cas7e and Cas8e proteins were developed upon aligning

916 the members of the corresponding protein families (Cas5e: pfam09704, TIGR1868, TIGR02593;

917 Cas6e: pfam08798, TIGR01907; Cas7e: pfam09344, TIGR01869; Cas8e: pfam 09481,

918 TIGR02547). A new HMM profile was generated for the less conserved Cse2 protein upon

919 aligning sequences with known 3D structure using PROMALS3D server (Pei et al., 2008) followed

920 by a series of iterative alignment/model building steps to include additional sequences and

921 increase sequence diversity. For the aligning processes of all five proteins, sequences were

922 dereplicated at 90\% identity using cd-hit (Huang et al., 2010) (with options -c 0.90 -g 1 -aS 0.9).

923 The dereplicated sequences were compared against each other using blastp from blast+ v2.6.0

924 (Altschul et al., 1990) with e-value 10e-05 and defaults for the rest of parameters. Hits were filtered

925 to retain those at $>=60 \%$ pairwise identity, and were next clustered using the mcl algorithm

926 (Enright et al., 2002) with inflation parameter of 2.0. Clusters with $>=10$ members were aligned

927 using Gismo (Neuwald and Liu, 2004) with default parameters, and consensus sequences were

928 extracted from the alignments. These consensus sequences, as well as singletons and

929 sequences from smaller clusters were aligned using Gismo (Neuwald and Liu, 2004). Alignments

930 were manually curated to remove shorter sequences that did not have one or more of the active

931 site positions and HMM profiles were generated using hmmbuild (Eddy, 2009). Hmmsearch

932 (Eddy, 2009) using the generated HMM profiles against all public genomes (isolates, SAGs, and

933 MAGs), and all public metagenomes resulted in hits which were subsequently aligned against the

934 generated HMM profiles. After selecting gene arrays that have all five complete or nearly complete

935 genes, we identified 6,964 arrays in public genomes and 5,000 arrays in public metagenomes.

936 Aligned sequences for all proteins from the same array were concatenated, and the resulting

937 sequences were dereplicated with cd-hit (Huang et al., 2010) at 90\% identity, aligned over at least

$93890 \%$ of the shorter sequences. This resulted in 2851 clusters, 1799 from metagenomes and 1052

939 from genomes. Whereas the alignment of the Cas8e proteins from these clusters showed high 
940 variability, the predicted L1 helix regions of the Cas8e, which have been shown to directly interact

941 with the PAM (Xiao et al., 2017), presented higher conservation. We generated a list with the L1

942 signatures from the dereplicated cluster set and we subsequently manually filtered out systems

943 that do not belong to known cultured mesophilic bacteria (Table S3). From the resulting list we

944 selected I-E CRISPR/Cas systems with a variety of L1 motifs for experimental validation with

945 PAM-DETECT.

946

947 Comparative analysis of I-B CAST transposases

948 We searched previous literature (Peters et al., 2017; Saito et al., 2021) for in silico identified I-B2

949 CASTs, which contain a fused tnsAB gene and are easily distinguished from I-B1 CASTs, which

950 contain separate tns $A$ and tns $B$ genes. We observed that one clade of the I-B2 CASTs

951 encompasses systems with tns $A B-t n s C-t n s D$ operons while having the tniQ gene separated,

952 whereas the other clade encompasses systems with tns $A B$-tns $C$-tniQ operons and the tns $D$ gene

953 separated. We denoted the systems in the former clade as I-B2.1 CASTs and in the latter clade

954 as I-B2.2 CASTs. We focused on the I-B2.2 CAST clade, that has no in vitro or in vivo

955 characterized members, and we discarded from further analysis the systems that lacked at least

956 one of the CRISPR-Cas or transposition genes (tns $A B, \operatorname{tns} C$, $t n s D$, tniQ, cas5, cas6, cas7, cas8).

957 We performed BlastP search (Altschul et al., 1990) using the TnsAB, TnsC, TnsD, TniQ proteins

958 of each selected I-B2.2 system as queries, aiming to identify additional I-B2.2 CAST candidates.

959 Our analysis yielded in total seven I-B2.2 systems and we selected six previously described I-

960 B2.1 systems for phylogenetic analysis (Saito et al., 2021). The alignment of I-B2.1 and I-B2.2

961 transposition proteins was performed using T-Coffee (Di Tommaso et al., 2011), the phylogenetic

962 trees were built using average distance and the BLOSUM62 matrix and they were visualized with

963 JalView (Waterhouse et al., 2009).

964

965 In silico analysis of RoCAST 
966 We predicted the CRISPR array of RoCAST by uploading the Rippkaea orientalis genomic region

967 between the Rocas5 and RotniQ to CRISPRFinder (Grissa et al., 2007). The RoCAST ends were

968 determined manually on Benchling by searching for repeat sequences of 20 nucleotides, with

969 maximum 5 mismatched nucleotides, within the Rippkaea orientalis genomic regions $1 \mathrm{~kb}$

970 upstream of the $R$. orientalis tns $A B$ and $1 \mathrm{~kb}$ downstream of the Rotns $D$. We identified two types

971 of repeat sequences present in both regions in opposite orientations and a candidate duplication

972 region. Notably, we identified five repeat sequences in the predicted left end region, with one of

973 the repeat sequences located downstream of the predicted duplication site, hence outside of the

974 predicted RoCAST limits. The TXTL transposition demonstrated that this repeat is not part of the

975 RoCAST transposon. 
Almendros, C., Guzmán, N.M., Díez-Villaseñor, C., García-Martínez, J., and Mojica, F.J.M. (2012). Target motifs affecting natural immunity by a constitutive CRISPR-Cas system in

979 Escherichia coli. PLoS One 7, e50797.

980

981

982

983

984

985

986

987

988

989

990

991

992

993

994

995

996

997

998

999

1000

1001

1002

1003

1004

1005

1006

1007

1008

1009

1010

1011

1012

Altschul, S.F., Gish, W., Miller, W., Myers, E.W., and Lipman, D.J. (1990). Basic local alignment search tool. J. Mol. Biol. 215, 403-410.

Barrangou, R., and Doudna, J.A. (2016). Applications of CRISPR technologies in research and beyond. Nature Biotechnology 34, 933-941.

Caliando, B.J., and Voigt, C.A. (2015). Targeted DNA degradation using a CRISPR device stably carried in the host genome. Nat. Commun. 6, 1-10.

Collias, D., and Beisel, C.L. (2021). CRISPR technologies and the search for the PAM-free nuclease. Nat. Commun. 12, 1-12.

Davidson, A.R., Lu, W.-T., Stanley, S.Y., Wang, J., Mejdani, M., Trost, C.N., Hicks, B.T., Lee, J., and Sontheimer, E.J. (2020). Anti-CRISPRs: protein inhibitors of CRISPR-Cas systems. Annu. Rev. Biochem. 89, 309-332.

Di Tommaso, P., Moretti, S., Xenarios, I., Orobitg, M., Montanyola, A., Chang, J.-M., Taly, J.-F., and Notredame, C. (2011). T-Coffee: a web server for the multiple sequence alignment of protein and RNA sequences using structural information and homology extension. Nucleic Acids Res. 39, W13-W17.

Eddy, S.R. (2009). A new generation of homology search tools based on probabilistic inference. Genome Inform. 23, 205-211.

Edgar, R., Domrachev, M., and Lash, A.E. (2002). Gene Expression Omnibus: NCBI gene expression and hybridization array data repository. Nucleic Acids Res. 30, 207-210.

Enright, A.J., Van Dongen, S., and Ouzounis, C.A. (2002). An efficient algorithm for large-scale detection of protein families. Nucleic Acids Res. 30, 1575-1584.

Fineran, P.C., Gerritzen, M.J.H., Suárez-Diez, M., Künne, T., Boekhorst, J., van Hijum, S.A.F.T., Staals, R.H.J., and Brouns, S.J.J. (2014). Degenerate target sites mediate rapid primed CRISPR adaptation. Proc. Natl. Acad. Sci. U. S. A. 111, E1629-E1638.

Fu, B.X.H., Wainberg, M., Kundaje, A., and Fire, A.Z. (2017). High-throughput characterization of Cascade type I-E CRISPR guide efficacy reveals unexpected PAM diversity and target sequence preferences. Genetics 206, 1727-1738.

Garamella, J., Marshall, R., Rustad, M., and Noireaux, V. (2016). The All E. coli TX-TL Toolbox 2.0: A platform for cell-free synthetic biology. ACS Synth. Biol. 5, 344-355.

Gasiunas, G., Young, J.K., Karvelis, T., Kazlauskas, D., Urbaitis, T., Jasnauskaite, M., Grusyte, M.M., Paulraj, S., Wang, P.-H., Hou, Z., et al. (2020). A catalogue of biochemically diverse CRISPR-Cas9 orthologs. Nat. Commun. 11, 5512.

Gomaa, A.A., Klumpe, H.E., Luo, M.L., Selle, K., Barrangou, R., and Beisel, C.L. (2014). 
1013

1014

1015

1016

1017

1018

1019

1020

1021

1022

1023

1024

1025

1026

1027

1028

1029

1030

1031

1032

1033

1034

1035

1036

1037

1038

1039

1040

1041

1042

1043

1044

1045

1046

1047

1048

1049

1050

Programmable removal of bacterial strains by use of genome-targeting CRISPR-Cas systems. mBio 5, e00928-13.

Grissa, I., Vergnaud, G., and Pourcel, C. (2007). CRISPRFinder: a web tool to identify clustered regularly interspaced short palindromic repeats. Nucleic Acids Res. 35, W52-W57.

Hidalgo-Cantabrana, C., and Barrangou, R. (2020). Characterization and applications of Type I CRISPR-Cas systems. Biochem. Soc. Trans. 48, 15-23.

Hochstrasser, M.L., Taylor, D.W., Kornfeld, J.E., Nogales, E., and Doudna, J.A. (2016). DNA targeting by a minimal CRISPR RNA-guided Cascade. Mol. Cell 63, 840-851.

Huang, Y., Niu, B., Gao, Y., Fu, L., and Li, W. (2010). CD-HIT Suite: a web server for clustering and comparing biological sequences. Bioinformatics 26, 680-682.

Huo, Y., Nam, K.H., Ding, F., Lee, H., Wu, L., Xiao, Y., Farchione, M.D., Jr, Zhou, S., Rajashankar, K., Kurinov, I., et al. (2014). Structures of CRISPR Cas3 offer mechanistic insights into Cascade-activated DNA unwinding and degradation. Nat. Struct. Mol. Biol. 21, 771-777.

Jiao, C., Sharma, S., Dugar, G., Peeck, N.L., Bischler, T., Wimmer, F., Yu, Y., Barquist, L., Schoen, C., Kurzai, O., et al. (2021). Noncanonical crRNAs derived from host transcripts enable multiplexable RNA detection by Cas9. Science 372, 941-948.

Jore, M.M., Lundgren, M., van Duijn, E., Bultema, J.B., Westra, E.R., Waghmare, S.P., Wiedenheft, B., Pul, U., Wurm, R., Wagner, R., et al. (2011). Structural basis for CRISPR RNAguided DNA recognition by Cascade. Nat. Struct. Mol. Biol. 18, 529-536.

Karvelis, T., Gasiunas, G., Young, J., Bigelyte, G., Silanskas, A., Cigan, M., and Siksnys, V. (2015). Rapid characterization of CRISPR-Cas9 protospacer adjacent motif sequence elements. Genome Biol. 16, 1-13.

Khakimzhan, A., Garenne, D., Tickman, B., Fontana, J., Carothers, J., and Noireaux, V. (2021). Complex dependence of CRISPR-Cas9 binding strength on guide RNA spacer lengths. Phys. Biol.

Klompe, S.E., Vo, P.L.H., Halpin-Healy, T.S., and Sternberg, S.H. (2019). Transposon-encoded CRISPR-Cas systems direct RNA-guided DNA integration. Nature 571, 219-225.

Leenay, R.T., and Beisel, C.L. (2017). Deciphering, communicating, and engineering the CRISPR PAM. J. Mol. Biol. 429, 177-191.

Leenay, R.T., Maksimchuk, K.R., Slotkowski, R.A., Agrawal, R.N., Gomaa, A.A., Briner, A.E., Barrangou, R., and Beisel, C.L. (2016). Identifying and visualizing functional PAM diversity across CRISPR-Cas systems. Mol. Cell 62, 137-147.

Li, M., Gong, L., Cheng, F., Yu, H., Zhao, D., Wang, R., Wang, T., Zhang, S., Zhou, J., Shmakov, S.A., et al. (2021). Toxin-antitoxin RNA pairs safeguard CRISPR-Cas systems. Science 372, eabe5601.

Liao, C., Slotkowski, R.A., Achmedov, T., and Beisel, C.L. (2019a). The Francisella novicida Cas12a is sensitive to the structure downstream of the terminal repeat in CRISPR arrays. RNA Biol. 16, 404-412. 
Liao, C., Ttofali, F., Slotkowski, R.A., Denny, S.R., Cecil, T.D., Leenay, R.T., Keung, A.J., and Beisel, C.L. (2019b). Modular one-pot assembly of CRISPR arrays enables library generation and reveals factors influencing crRNA biogenesis. Nat. Commun. 10, 2948. and its application for genetic manipulations. Curr. Issues Mol. Biol. 26, 1-14.

Makarova, K.S., Wolf, Y.I., Alkhnbashi, O.S., Costa, F., Shah, S.A., Saunders, S.J., Barrangou, R., Brouns, S.J.J., Charpentier, E., Haft, D.H., et al. (2015). An updated evolutionary classification of CRISPR-Cas systems. Nat. Rev. Microbiol. 13, 722-736.

Makarova, K.S., Wolf, Y.I., Iranzo, J., Shmakov, S.A., Alkhnbashi, O.S., Brouns, S.J.J.,

Charpentier, E., Cheng, D., Haft, D.H., Horvath, P., et al. (2019). Evolutionary classification of CRISPR-Cas systems: a burst of class 2 and derived variants. Nat. Rev. Microbiol. 18, 67-83.

Marino, N.D., Zhang, J.Y., Borges, A.L., Sousa, A.A., Leon, L.M., Rauch, B.J., Walton, R.T., V CRISPR-Cas inhibitors. Science 362, 240-242.

Marshall, R., Maxwell, C.S., Collins, S.P., Jacobsen, T., Luo, M.L., Begemann, M.B., Gray, B.N., January, E., Singer, A., He, Y., et al. (2018). Rapid and scalable characterization of CRISPR technologies using an E. coli cell-free transcription-translation system. Mol. Cell 69, 1461068 157.e3.

Maxwell, C.S., Jacobsen, T., Marshall, R., Noireaux, V., and Beisel, C.L. (2018). A detailed cellMethods 143, 48-57.

Mulepati, S., and Bailey, S. (2013). In vitro reconstitution of an Escherichia coli RNA-guided immune system reveals unidir
Chem. 288, 22184-22192.

Musharova, O., Sitnik, V., Vlot, M., Savitskaya, E., Datsenko, K.A., Krivoy, A., Fedorov, I., Semenova, E., Brouns, S.J.J., and Severinov, K. (2019). Systematic analysis of Type I-E Escherichia coli CRISPR-Cas PAM sequences ability to promote interference and primed adaptation. Mol. Microbiol. 111, 1558-1570.

Neuwald, A.F., and Liu, J.S. (2004). Gapped alignment of protein sequence motifs through Monte Carlo optimization of a hidden Markov model. BMC Bioinformatics 5.

Pausch, P., Müller-Esparza, H., Gleditzsch, D., Altegoer, F., Randau, L., and Bange, G. (2017). Structural variation of Type I-F CRISPR RNA guided DNA surveillance. Mol. Cell 67, 622632.e4.

1084 Pei, J., Kim, B.-H., and Grishin, N.V. (2008). PROMALS3D: a tool for multiple protein sequence 1085 and structure alignments. Nucleic Acids Res. 36, 2295-2300.

Petassi, M.T., Hsieh, S.-C., and Peters, J.E. (2020). Guide RNA categorization enables target site choice in Tn7-CRISPR-Cas transposons. Cell 183, 1757-1771.e18.

Peters, J.E., Makarova, K.S., Shmakov, S., and Koonin, E.V. (2017). Recruitment of CRISPRCas systems by Tn7-like transposons. Proc. Natl. Acad. Sci. U. S. A. 114, E7358-E7366. 
Pickar-Oliver, A., and Gersbach, C.A. (2019). The next generation of CRISPR-Cas technologies and applications. Nat. Rev. Mol. Cell Biol. 20, 490-507. system reveals distinct dynamics of spacer acquisition and loss. RNA 23, 1525-1538.

1097 Rollins, M.F., Schuman, J.T., Paulus, K., Bukhari, H.S.T., and Wiedenheft, B. (2015).

1100 Saito, M., Ladha, A., Strecker, J., Faure, G., Neumann, E., Altae-Tran, H., Macrae, R.K., and Zhang, F. (2021). Dual modes of CRISPR-associated transposon homing. Cell 184, 24411102 2453.e18.

1103 Shin, J., and Noireaux, V. (2012). An E. coli cell-free expression toolbox: application to synthetic gene circuits and artificial cells. ACS Synth. Biol. 1, 29-41.

Silas, S., Lucas-Elio, P., Jackson, S.A., Aroca-Crevillén, A., Hansen, L.L., Fineran, P.C., Fire, A.Z., and Sánchez-Amat, A. (2017). Type III CRISPR-Cas systems can provide redundancy to counteract viral escape from type I systems. Elife 6, e27601.

Silverman, A.D., Karim, A.S., and Jewett, M.C. (2020). Cell-free gene expression: an expanded repertoire of applications. Nat. Rev. Genet. 21, 151-170.

Sinkunas, T., Gasiunas, G., Waghmare, S.P., Dickman, M.J., Barrangou, R., Horvath, P., and

1113 Stern, A., Keren, L., Wurtzel, O., Amitai, G., and Sorek, R. (2010). Self-targeting by CRISPR: gene regulation or autoimmunity? Trends Genet. 26, 335-340.

1115 Strecker, J., Ladha, A., Gardner, Z., Schmid-Burgk, J.L., Makarova, K.S., Koonin, E.V., and Zhang, F. (2019). RNA-guided DNA insertion with CRISPR-associated transposases. Science $1117365,48-53$.

1118 Tay, M., Liu, S., and Yuan, Y.A. (2015). Crystal structure of Thermobifida fusca Cse1 reveals target DNA binding site. Protein Sci. 24, 236-245.

Tuminauskaite, D., Norkunaite, D., Fiodorovaite, M., Tumas, S., Songailiene, I., Tamulaitiene, G., and Sinkunas, T. (2020). DNA interference is controlled by R-loop length in a type I-F1 CRISPR-Cas system. BMC Biol. 18, 65.

1127 Vo, P.L.H., Ronda, C., Klompe, S.E., Chen, E.E., Acree, C., Wang, H.H., and Sternberg, S.H. 1128 (2021). CRISPR RNA-guided integrases for high-efficiency, multiplexed bacterial genome 
engineering. Nat. Biotechnol. 39, 480-489.

Wandera, K.G., Collins, S.P., Wimmer, F., Marshall, R., Noireaux, V., and Beisel, C.L. (2020). An enhanced assay to characterize anti-CRISPR proteins using a cell-free transcriptiontranslation system. Methods 172, 42-50.

Waterhouse, A.M., Procter, J.B., Martin, D.M.A., Clamp, M., and Barton, G.J. (2009). Jalview Version 2--a multiple sequence alignment editor and analysis workbench. Bioinformatics 25, $1189-1191$.

Watters, K.E., Fellmann, C., Bai, H.B., Ren, S.M., and Doudna, J.A. (2018). Systematic discovery of natural CRISPR-Cas12a inhibitors. Science 362, 236-239.

Westra, E.R., van Erp, P.B.G., Künne, T., Wong, S.P., Staals, R.H.J., Seegers, C.L.C., Bollen, S., Jore, M.M., Semenova, E., Severinov, K., et al. (2012). CRISPR immunity relies on the consecutive binding and degradation of negatively supercoiled invader DNA by Cascade and Cas3. Mol. Cell 46, 595-605.

Wimmer, F., and Beisel, C.L. (2019). CRISPR-Cas systems and the paradox of self-targeting spacers. Front. Microbiol. 10, 3078.

Xiao, Y., Luo, M., Hayes, R.P., Kim, J., Ng, S., Ding, F., Liao, M., and Ke, A. (2017). Structure basis for directional R-loop formation and substrate handover mechanisms in type I CRISPRCas system. Cell 170, 48-60.e11.

Xue, C., Seetharam, A.S., Musharova, O., Severinov, K., Brouns, S.J.J., Severin, A.J., and Sashital, D.G. (2015). CRISPR interference and priming varies with individual spacer sequences. Nucleic Acids Res. 43, 10831-10847.

Yin, Y., Yang, B., and Entwistle, S. (2019). Bioinformatics identification of anti-CRISPR loci by using homology, guilt-by-association, and CRISPR self-targeting spacer approaches. mSystems 4, e00455-19.

1153 Zetsche, B., Abudayyeh, O.O., Gootenberg, J.S., Scott, D.A., and Zhang, F. (2020). A survey of 1154 genome editing activity for 16 Cas12a orthologs. Keio J. Med. 69, 59-65.

Zheng, Y., Han, J., Wang, B., Hu, X., Li, R., Shen, W., Ma, X., Ma, L., Yi, L., Yang, S., et al. (2019). Characterization and repurposing of the endogenous Type I-F CRISPR-Cas system of Zymomonas mobilis for genome engineering. Nucleic Acids Research 47, 11461-11475. Front Bioeng Biotechnol 8, 62. 